\title{
DISCREPANCY AND EIGENVALUES OF CAYLEY GRAPHS
}

\author{
YOSHIHARU KOHAYAKAWA, VOJTĚCH RÖDL, AND MATHIAS SCHACHT \\ Dedicated to the memory of Professor Miroslav Fiedler
}

\begin{abstract}
We consider quasirandom properties for Cayley graphs of finite abelian groups. We show that having uniform edge-distribution (i.e., small discrepancy) and having large eigenvalue gap are equivalent properties for such Cayley graphs, even if they are sparse. This positively answers a question of Chung and Graham ["Sparse quasi-random graphs", Combinatorica 22 (2002), no. 2, 217-244] for the particular case of Cayley graphs of abelian groups, while in general the answer is negative.
\end{abstract}

\section{§1. INTRODUCTION}

Professor Miroslav Fiedler discovered a very fruitful relationship between connectivity properties of graphs and their spectra. Among other things, his works [14,15] from the 1970s, together with other pioneering work $[12,13,18]$, gave birth to what is now known as spectral partitioning of graphs. Fiedler considered the so called combinatorial Laplacian $L(G)$ of graphs $G$ and their spectrum $0=\lambda_{1} \leqslant \lambda_{2} \leqslant \cdots \leqslant \lambda_{n}(n=|V(G)|)$. Generalizing the fact that $G$ is connected if and only if $\lambda_{2}>0$, Fiedler named $\lambda_{2}$ the algebraic connectivity of $G$ and went on to prove that $\lambda_{2}$ is a lower bound for the standard connectivity of $G$ (unless $G$ is the complete graph). Furthermore, he also considered partitioning the vertex set of $G$ by considering the coordinates of the eigenvector belonging to $\lambda_{2}$. The algebraic connectivity of a graph is now sometimes referred to as the Fiedler value and the associated eigenvector is referred to as the Fiedler vector. Alon [1] and Sinclair and Jerrum [26] later proved that graphs with small Fiedler value can be partitioned according to the Fiedler vector in a direct way to produce a cut that is small in relative terms (that is, in terms of the ratio of the number of cut edges to the number of separated vertices).

Date: March 1, 2022.

2010 Mathematics Subject Classification. Primary: 05C50. Secondary: 05C80.

Key words and phrases. Eigenvalues, discrepancy, quasirandomness, Cayley graphs.

The first author was supported by FAPESP (2013/03447-6, 2013/07699-0), CNPq (459335/2014-6, 310974/2013-5) and Project MaCLinC/USP. The second author was supported by NSF grant DMS 1301698. The third author was supported through the Heisenberg-Programme of the DFG. The collaboration of the first and third authors is supported by CAPES/DAAD PROBRAL project 430/15. 
While a small Fiedler value tells us that the graph in question may be split along a "small cut", a large Fiedler value implies that the graph is an expander, that is, it has no cuts that are "small" [3,29]. In this paper, we investigate the relation between such "edge-distribution properties" and spectra, but focusing on the case of "uniform edge-distribution", by which we mean the quasirandom case, in the sense of Chung, Graham and Wilson [9]. ${ }^{1}$ Since we shall be concerned with Cayley graphs, which are regular graphs, for simplicity, we shall work with adjacency matrices and not with combinatorial Laplacians.

Let an $n$-vertex graph $G$ be given. The eigenvalues of $G$ are simply the eigenvalues of the $n$ by $n, 0-1$ adjacency matrix of $G$, with 1 indicating edges. Let $\lambda_{k}=\lambda_{k}(G)$ be the $k$ th largest eigenvalue of $G$, in absolute value. Recall that $G$ is said to be "quasirandom" if the edges of $G$ are "uniformly distributed" (we postpone the precise definition; see Definition 1.1). A fundamental result relating the $\lambda_{i}$ to quasirandomness states that there is a large gap between $\lambda_{1}$ and $\lambda_{k}(k \geqslant 2)$ if and only if $G$ is quasirandom.

The assertion above may be turned precise in different ways. We are interested in the form given by Chung, Graham, and Wilson [9]. Recall that [9] presents a "theory of quasirandomness" for graphs, exhibiting several, quite disparate almost sure properties of graphs that are, quite surprisingly, equivalent in a deterministic sense. Earlier work in this direction is due to Thomason [30] (see also [31]), and also Alon [1], Alon and Chung [2], Frankl, Rödl and Wilson [16], and Rödl [24]. One of the so-called "quasirandom properties" that is presented in [9] is the "eigenvalue gap" between $\lambda_{1}$ and $\lambda_{k}(k \geqslant 2)$.

Chung and Graham [8] set out to investigate the extension of the results in [9] to sparse graphs, that is, graphs with vanishing edge-density. As it turns out, a naïve approach to such a project is doomed to fail, as the results in [9] do not generalize to the "sparse case" in the expected manner (for a thorough discussion on this point, the interested reader is referred to [8] and also to [4,7,10,19-21]). In particular, having succeeded in proving that eigenvalue gap does imply uniform distribution of edges in the sparse case, Chung and Graham asked whether the converse also holds (see [8, p. 230]). An affirmative answer to this question would fully generalize the relationship between these two concepts to the sparse case.

However, Krivelevich and Sudakov [21] showed that the answer to the question posed by Chung and Graham is negative, by constructing a suitable family of counterexamples. Here, our aim is to show that the answer is positive if one considers Cayley graphs of finite abelian groups, regardless of the density of the graph. It is worth noting that several

\footnotetext{
${ }^{1}$ Owing to this focus, spectral graph partitioning will not be discussed here; the interested reader is referred to, e.g., Spielman [27] and Spielman and Teng [28].
} 
explicit constructions of quasirandom graphs are indeed Cayley graphs (see, e.g., [31] and [21, Section 3]).

We use the following notation. If $G=(V, E)$ is a graph, we write $e(G)$ for the number of edges $|E|$ in $G$. If $U \subset V$ is a set of vertices of $G$, then $G[U]$ denotes the subgraph of $G$ induced by $U$. Furthermore, if $W \subset V$ is disjoint from $U$, then we write $G[U, W]$ for the bipartite subgraph of $G$ naturally induced by the pair $(U, W)$. We also sometimes write $E(U, W)=E_{G}(U, W)$ for the edge set of $G[U, W]$.

If $\delta>0$, we write $x \sim_{\delta} y$ to mean that

$$
(1-\delta) y \leqslant x \leqslant(1+\delta) y
$$

Moreover, sometimes it will be convenient to write $O_{1}(\delta)$ for any term $\beta$ that satisfies $|\beta| \leqslant \delta$. Observe that, clearly $x \sim_{\delta} y$ is equivalent to $x=\left(1+O_{1}(\delta)\right) y$.

Definition $1.1(\mathrm{DISC}(\delta))$. Let $0<\delta \leqslant 1$ be given. We say that an $n$-vertex graph $G$ $(n \geqslant 2)$ satisfies property $\operatorname{DISC}(\delta)$ if the following assertion holds: for all $U \subset V(G)$ with $|U| \geqslant \delta n$, we have

$$
e_{G}(U)=e(G[U]) \sim_{\delta} e(G)\left(\begin{array}{c}
|U| \\
2
\end{array}\right) /\left(\begin{array}{l}
n \\
2
\end{array}\right) .
$$

The following concept of DISC $_{2}$ is very much related to DISC, as we shall see next.

Definition $1.2\left(\operatorname{DISC}_{2}\left(\delta^{\prime}\right)\right)$. Let $0<\delta^{\prime} \leqslant 1$ be given. We say that an $n$-vertex graph $G$ $(n \geqslant 2)$ satisfies property $\mathrm{DISC}_{2}\left(\delta^{\prime}\right)$ if the following assertion holds: for all disjoint $U$ and $W \subset V(G)$ with $|U|,|W| \geqslant \delta^{\prime} n$, we have

$$
e_{G}(U, W)=e(G[U, W]) \sim_{\delta^{\prime}} e(G)|U||W| /\left(\begin{array}{l}
n \\
2
\end{array}\right) .
$$

The following fact is very easy to prove and we omit its proof.

Fact 1.3. For any $0<\delta^{\prime} \leqslant 1$, there is $0<\delta=\delta\left(\delta^{\prime}\right) \leqslant 1$ such that any graph that satisfies $\mathrm{DISC}(\delta)$ must also satisfy $\mathrm{DISC}_{2}\left(\delta^{\prime}\right)$.

Given a graph $G$, let $\mathbf{A}=\left(a_{u v}\right)_{u, v \in V(G)}$ be the $0-1$ adjacency matrix of $G$, with 1 denoting edges. The eigenvalues of $G$ are simply the eigenvalues of $\mathbf{A}$. Since $\mathbf{A}$ is symmetric, its eigenvalues are real. As usual, we adjust the notation so that these eigenvalues are such that

$$
\lambda_{1} \geqslant\left|\lambda_{2}\right| \geqslant \cdots \geqslant\left|\lambda_{n}\right|
$$

(the fact that $\lambda_{1} \geqslant 0$ follows, for instance, from the fact that the sum of the $\lambda_{i}$ is equal to the trace of $\mathbf{A}$, which is 0 ). 
Definition $1.4(\operatorname{EIG}(\varepsilon))$. Let $0<\varepsilon \leqslant 1$ be given. We say that an $n$-vertex graph $G$ satisfies property $\operatorname{EIG}(\varepsilon)$ if the following holds. Let $\bar{d}=\bar{d}(G)=2 e(G) / n$ be the average degree of $G$, and let $\lambda_{1}, \ldots, \lambda_{n}$ be the eigenvalues of $G$, with the notation adjusted in such a way that (1) holds. Then

(i) $\lambda_{1} \sim_{\varepsilon} \bar{d}$,

(ii) $\left|\lambda_{i}\right| \leqslant \varepsilon \bar{d}$ for all $1<i \leqslant n$.

Finally, we define Cayley graphs.

Definition 1.5 (Cayley graph $G(\Gamma, A)$ ). Let $\Gamma$ be an abelian group and let $A \subset \Gamma \backslash\{0\}$ be symmetric, that is, $A=-A$. The Cayley graph $G=G(\Gamma, A)$ is defined to be the graph on $\Gamma$, with two vertices $\gamma$ and $\gamma^{\prime} \in \Gamma$ adjacent in $G$ if and only if $\gamma^{\prime}-\gamma \in A$.

We only consider finite graphs and finite abelian groups. The main aim is to answer a question of Chung and Graham from [8] in the positive for an interesting class of graphs.

Theorem 1.6. For every $\varepsilon>0$, there exist $\delta>0$ and $n_{0}$ such that the following holds. Let $G=G(\Gamma, A)$ be a Cayley graph for some abelian group $\Gamma$ with $n=|\Gamma| \geqslant n_{0}$ elements and a symmetric set $A=-A \subseteq \Gamma \backslash\{0\}$. If $G$ satisfies property $\operatorname{DISC}(\delta)$, then $G$ satisfies $\operatorname{EIG}(\varepsilon)$.

The proof of this theorem is given in Section 2. We close this introduction with a few remarks concerning Theorem 1.6.

We first observe that Theorem 1.6, together with the results of Chung and Graham [8], imply that properties DISC and EIG are equivalent for Cayley graphs. More precisely, by DISC implies EIG for Cayley graphs we mean the following: for every $\varepsilon>0$ there is a $\delta=\delta(\varepsilon)>0$ such that, for any sequence of positive integers $\left(n_{k}\right)_{k}$ with $n_{k} \rightarrow \infty$ as $k \rightarrow \infty$, and any sequence $\left(G_{k}\right)_{k}$ of Cayley graphs with $\left|V\left(G_{k}\right)\right|=n_{k}$, we have that if all but finitely many graphs $G_{k}$ satisfy $\operatorname{DISC}(\delta)$, then all but finitely many $G_{k}$ satisfy EIG $(\varepsilon)$. Theorem 1.6 tells us that DISC implies EIG for sequences of Cayley graphs. In $[8$, Theorem 1] it is proved that EIG implies DISC in the same sense for sequences of arbitrary graphs with average degree tending to infinity. This establishes the equivalence of the properties DISC and EIG for Cayley graphs with diverging average degree.

Secondly, we note that in general it is not true that DISC implies EIG for arbitrary sequences of graphs. This was already pointed out by Krivelevich and Sudakov in [21]. For every $\varepsilon>0$ and every $\delta>0$, they constructed an infinite sequence of graphs that satisfy $\operatorname{DISC}(\delta)$ but fail to satisfy $(i)$ in the definition of $\operatorname{EIG}(\varepsilon)$ (see Definition 1.4).

The following example is a different probabilistic construction: For $p=p(n) \rightarrow 0$ with $p n \gg 1$ as $n \rightarrow \infty$, consider the graph $G$ given by the union of the random graph 
$G(n, p)$ and a disjoint clique of size $\alpha p n$ for some constant $\alpha>0$. Such a graph $G$ has density $(1+o(1)) p$ and for every fixed $\delta>0$ with high probability it satisfies $\operatorname{DISC}(\delta)$. However, $\alpha p n-1$ is one of the eigenvalues of its adjacency matrix and, hence, $G$ fails to satisfy $(i i)$ in the definition of $\operatorname{EIG}(\varepsilon)$ for any fixed $\varepsilon \in(0, \alpha)$.

We also remark that in [8], it is proved that, under some additional conditions, DISC implies EIG for sequences of sparse graphs. This additional assumption combined with DISC implies that almost every graph in the sequence contains the "expected number" of closed walks of length $\ell$ for some even $\ell \geqslant 4$. More precisely, for a sequence of graphs $G_{n}$ with average degree $\bar{d}_{n}$ we say it satisfies $\operatorname{CIRCUIT}_{\ell}$ if the number of closed walks of length $\ell$ in $G_{n}$ is $(1+o(1))\left(\bar{d}_{n}\right)^{\ell}$. We remark that Theorem 1.6 is not a consequence of the result of Chung and Graham, since there exist sequences of Cayley graphs satisfying DISC, and hence by Theorem 1.6 also EIG, but fail to have CIRCUIT $_{\ell}$ for any fixed even $\ell \geqslant 4$. We next sketch the construction of such a sequence.

Let

$$
p=p(n)=\frac{\log ^{2} n}{n}
$$

and consider the random cyclic Cayley graph $\mathcal{C}_{n, p}=G(\mathbb{Z} / n \mathbb{Z}, A)$, where independently for every $a \in(\mathbb{Z} / n \mathbb{Z}) \backslash\{0\}$ both elements $a$ and $-a$ are included in $A$ with probability $p / 2$. It follows from standard Chernoff-type estimates that asymptotically almost surely $\mathcal{C}_{n, p}$ satisfies DISC and has average degree $\bar{d}_{n}=(1+o(1) p n$. Consequently, by Theorem 1.6 it also satisfies EIG.

On the other hand, owing to the choice of $p$ we have

$$
p n^{2} \gg(p n)^{\ell}
$$

for every fixed even $\ell \geqslant 4$ and sufficiently large $n$. Hence, for every even $\ell \geqslant 4$ in expectation the number of "degenerated walks" which only use one edge is $\gg\left(\bar{d}_{n}\right)^{\ell}$. This implies that with positive probability $\mathcal{C}_{n, p}$ satisfies DISC and EIG, but fails to satisfy $\mathrm{CIRCUIT}_{\ell}$ for every even $\ell \geqslant 4$. Using appropriate blowups of such graphs yields sequences of Cayley graphs with these properties for any density $p$ with $\log ^{2} n / n \ll p \ll 1$.

Finally, we remark that very recently Conlon and Zhao [11] extended Theorem 1.6 for Cayley graphs for arbitrary (not necessarily abelian) finite groups.

Acknowledgements. The proof of Theorem 1.6 presented here is based on an idea of Tim Gowers [17]. The authors proved this result with a longer combinatorial argument, which we include in the appendix. On learning about the result, Tim Gowers suggested the alternative, elegant proof given below. We are grateful to him for letting us include his proof here. 


\section{§2. Proof of the main Result}

2.1. Eigenvalues of Cayley graphs of abelian groups. Theorem 2.1 below tells us how to compute the eigenvalues of Cayley graphs of abelian groups (Theorem 2.1 follows from a more general result due to Lovász [22]; see also [23, Exercise 11.8] and [6]).

Before we state Theorem 2.1, we recall some basic facts about group characters (for more details see, e.g., Serre [25]). Let $\Gamma$ be a finite abelian group. In this case, an irreducible character $\chi$ of $\Gamma$ may be viewed as a group homomorphism $\chi: \Gamma \rightarrow S^{1}$, i.e., $\chi(a+b)=\chi(a) \chi(b)$ for all $a, b \in \Gamma$, where $S^{1}$ is the multiplicative group of complex numbers of absolute value 1 . If $\Gamma$ has order $n$, then there are $n$ irreducible characters, say, $\chi_{1}, \ldots, \chi_{n}$, and these characters satisfy the following orthogonality property:

$$
\left\langle\chi_{i}, \chi_{j}\right\rangle=\sum_{\gamma \in \Gamma} \chi_{i}(\gamma) \chi_{j}(\gamma)=0
$$

for all $i \neq j$. These facts and a simple computation suffice to prove the following well known result, the short proof of which we include for completeness. We shall use the following notation: if $X$ is a set, we also write $X$ for the $\{0,1\}$-indicator function of $X$, so that $X(a)=1$ if $a \in X$ and $X(a)=0$ otherwise.

Theorem 2.1. Let $G=G(\Gamma, A)$ be a Cayley graph for some finite abelian group $\Gamma$ and a symmetric set $A=-A \subseteq \Gamma \backslash\{0\}$. For any character $\chi: \Gamma \rightarrow S^{1}$ of $\Gamma$, put

$$
\lambda^{(\chi)}=\langle A, \chi\rangle=\sum_{a \in A} \chi(a) .
$$

Then the eigenvalues of $G$ are the $\lambda^{(\chi)}$, where $\chi$ runs over all $n=|\Gamma|$ irreducible characters of $\Gamma$.

Proof. Let $\chi: \Gamma \rightarrow S^{1}$ be an irreducible character of $\Gamma$. Let $\lambda^{(\chi)}$ be as defined in (3). Consider the vector $\mathbf{v}^{(\chi)}=(\chi(\gamma))_{\gamma \in \Gamma}^{T}$, with entries indexed by the elements of $\Gamma=V(G)$. Let $\mathbf{A}=\left(a_{\gamma \gamma^{\prime}}\right)_{\gamma, \gamma^{\prime} \in \Gamma}$ be the adjacency matrix of $G$.

Fix $\gamma \in \Gamma$. Observe that the $\gamma$-entry $\left(\mathbf{A v}^{(\chi)}\right)_{\gamma}$ of the vector $\mathbf{A v}^{(\chi)}$ is

$$
\left(\mathbf{A v}^{(\chi)}\right)_{\gamma}=\sum_{a \in A} \chi(\gamma-a)=\sum_{a \in A} \chi(\gamma+a)=\left(\sum_{a \in A} \chi(a)\right) \chi(\gamma)=\lambda^{(\chi)} \chi(\gamma)
$$

and hence $\mathbf{A} \mathbf{v}^{(\chi)}=\lambda^{(\chi)} \mathbf{v}^{(\chi)}$; that is, $\mathbf{v}^{(\chi)}$ is an eigenvector of $\mathbf{A}$ with eigenvalue $\lambda^{(\chi)}$.

Let $\chi_{j}: \Gamma \rightarrow S^{1}(1 \leqslant j \leqslant n)$ be the irreducible characters of $\Gamma$ and set $\mathbf{v}_{j}=\mathbf{v}^{\left(\chi_{j}\right)}$ for all $1 \leqslant j \leqslant n$. By $(2),\left\langle\mathbf{v}_{j}, \mathbf{v}_{j^{\prime}}\right\rangle=0$ if $j \neq j^{\prime}$. Therefore, the $\mathbf{v}_{j}(1 \leqslant j \leqslant n)$ form an orthogonal basis of eigenvectors of the matrix $\mathbf{A}$ and, hence, $\lambda^{\left(\chi_{j}\right)}(j=1, \ldots, n)$ are indeed all the eigenvalues of $G$. 
Remark 2.2. The eigenvalue $\lambda_{1}=d=|A|$ may be obtained from (3) by letting $\chi$ be the trivial character $\chi(x)=1$ for all $x \in \Gamma$.

2.2. The proof. We shall prove that $\neg \operatorname{EIG}(\varepsilon) \Rightarrow \neg \operatorname{DISC}(\delta)$. By Theorem 2.1 and Remark 2.2, our assumption implies that there is a character $\chi \not \equiv 1$ such that

$$
\left|\lambda^{(\chi)}\right|=|\langle A, \chi\rangle| \geqslant \varepsilon|A| .
$$

We shall fix this $\chi$ and we shall use it to construct sets $X$ and $Y \subset V(G)$ that "witness" the fact that $\neg \operatorname{DISC}(\delta)$ holds.

First we introduce some notation. Let $0 \leqslant \chi_{\mathrm{ARG}}(\gamma)<2 \pi$ be defined by $\chi(\gamma)=\mathrm{e}^{\mathrm{i} \chi_{\mathrm{ARG}}(\gamma)}$. For $\gamma \in \Gamma$, let

$$
c(\gamma)=\operatorname{Re}(\chi(\gamma))=\cos \left(\chi_{\mathrm{ARG}}(\gamma)\right)
$$

and

$$
s(\gamma)=\operatorname{Im}(\chi(\gamma))=\sin \left(\chi_{\mathrm{ARG}}(\gamma)\right) .
$$

Applying the orthogonality relation (2) to $\chi$ and the trivial character $\chi \equiv 1$, denoted below by 1 , gives us that

$$
0=\langle\mathbf{1}, \chi\rangle=\sum_{\gamma \in \Gamma} \mathrm{e}^{\mathrm{i} \chi_{\mathrm{ARG}}(\gamma)}=\sum_{\gamma \in \Gamma}(c(\gamma)+\mathrm{i} s(\gamma)) .
$$

Consequently,

$$
\sum_{\gamma \in \Gamma} c(\gamma)=\sum_{\gamma \in \Gamma} s(\gamma)=0 .
$$

Given two functions $f$ and $g: \Gamma \rightarrow \mathbb{C}$, let $f * g: \Gamma \rightarrow \mathbb{C}$ be their convolution, given by

$$
(f * g)(\alpha)=\sum_{\gamma \in \Gamma} f(\alpha-\gamma) g(\gamma) .
$$

In what follows, we let $m$ be the cardinality of the image of $\chi$ :

$$
m=|\{\chi(\gamma): \gamma \in \Gamma\}| .
$$

Since $\chi \not \equiv 1$, we have $m>1$. We shall need the following fact.

Lemma 2.3. We have

$$
\sum_{\gamma \in \Gamma} c^{2}(\gamma)= \begin{cases}n & \text { if } m=2 \\ n / 2 & \text { if } m>2\end{cases}
$$


(ii)

$$
\begin{aligned}
\left\langle A, \frac{1}{2}(1+c) * \frac{1}{2}(1+c)\right\rangle & =\frac{1}{4} n|A|+\frac{1}{4}\langle A, c * c\rangle \\
& = \begin{cases}\frac{1}{4} n|A|+\frac{1}{4} n\langle A, c\rangle & \text { if } m=2 \\
\frac{1}{4} n|A|+\frac{1}{8} n\langle A, c\rangle & \text { if } m>2 .\end{cases}
\end{aligned}
$$

We postpone the proof of Lemma 2.3 to Section 2.3, and proceed to prove our main theorem. Let $-X$ and $Y \subset \Gamma$ be generated at random as follows: we include $\gamma \in \Gamma$ in $-X$ with probability $p(\gamma)=(1+c(\gamma)) / 2$ and we include $\gamma \in \Gamma$ in $Y$ with the same probability $p(\gamma)$. with all these events independent.

By (5) we have $\sum_{\gamma \in \Gamma} p(\gamma)=n / 2$. Therefore, by a Chernoff type inequality (see, e.g., Alon and Spencer [5, Theorem A.1.4]), we have

$$
\mathbb{P}\left(|X|=\left(\frac{1}{2}+o(1)\right) n\right)=1-o(1)
$$

and

$$
\mathbb{P}\left(|Y|=\left(\frac{1}{2}+o(1)\right) n\right)=1-o(1) .
$$

In view of Lemma $2.3(i)$, we have

$$
\sum_{\gamma \in \Gamma} p(-\gamma) p(\gamma)=\sum_{\gamma \in \Gamma} p^{2}(\gamma)=\frac{1}{4} \sum_{\gamma \in \Gamma}(1+c(\gamma))^{2} \stackrel{(5)}{=} \frac{1}{4} n+\frac{1}{4} \sum_{\gamma \in \Gamma} c(\gamma)^{2}=\frac{3}{8} n
$$

if $m>2$ and $\sum_{\gamma \in \Gamma} p(-\gamma) p(\gamma)=n / 2$ if $m=2$. Consequently, if $m>2$, we have

$$
\mathbb{P}\left(|X \cap Y|=\left(\frac{3}{8}+o(1)\right) n\right)=1-o(1)
$$

and hence, in view of (8) and (9), we have

$$
\mathbb{P}\left(|X \cup Y|=\left(\frac{5}{8}+o(1)\right) n\right)=1-o(1) .
$$

Similarly, if $m=2$, we have

$$
\mathbb{P}\left(|X \cap Y|=\left(\frac{1}{2}+o(1)\right) n\right)=1-o(1)
$$

and

$$
\mathbb{P}\left(|X \cup Y|=\left(\frac{1}{2}+o(1)\right) n\right)=1-o(1) .
$$

On the other hand, in view of our assumption (4) and $A=-A$ we have

$$
\varepsilon|A| \leqslant|\langle A, \chi\rangle|=|\langle A, c\rangle| .
$$


DISCREPANCY AND EIGENVALUES

Recall that $p(\gamma)=(1+c(\gamma)) / 2$ is the probability that we include $\gamma$ in $-X$ and in $Y$. By the linearity of the expectation and the independence, we have ${ }^{2}$

$$
\begin{aligned}
\mathbb{E}(\langle A,(-X) * Y\rangle) & =\mathbb{E}\left(\sum_{a \in A} \sum_{\gamma \in \Gamma}(-X)(a-\gamma) Y(\gamma)\right) \\
& =\sum_{a \in A} \sum_{\gamma \in \Gamma} \mathbb{E}((-X)(a-\gamma)) \mathbb{E}(Y(\gamma))=\sum_{a \in A} \sum_{\gamma \in \Gamma} p(a-\gamma) p(\gamma) \\
& =\left\langle A, \frac{1}{2}(1+c) * \frac{1}{2}(1+c)\right\rangle .
\end{aligned}
$$

By Lemma $2.3(i i)$, we thus have

$$
\left|\mathbb{E}(\langle A,(-X) * Y\rangle)-\frac{1}{4} n\right| A|| \geqslant \frac{1}{8} n|\langle A, c\rangle| \geqslant \frac{1}{8} \varepsilon n|A| .
$$

On the other hand,

$$
\langle A,(-X) * Y\rangle=\sum_{a \in A} \sum_{\gamma \in \Gamma}(-X)(a-\gamma) Y(\gamma)=\sum_{a \in A} \sum_{\gamma \in \Gamma} X(-a+\gamma) Y(\gamma)=e(X, Y),
$$

with the edges in $X \cap Y$ counted twice. Since $0 \leqslant e(X, Y) \leqslant n|A|$, the random variable

$$
\eta=\eta(X, Y)=\langle A,(-X) * Y\rangle-\frac{1}{4} n|A|=e(X, Y)-\frac{1}{4} n|A|
$$

satisfies

$$
-\frac{1}{4} n|A| \leqslant \eta \leqslant \frac{3}{4} n|A|
$$

Let $q$ be the probability that $|\eta| \leqslant \varepsilon n|A| / 16$. Then, by (14) and (15),

$$
\frac{1}{8} \varepsilon n|A| \leqslant|\mathbb{E}(\eta)| \leqslant \mathbb{E}(|\eta|) \leqslant \frac{1}{16} \varepsilon n|A| q+\frac{3}{4} n|A|(1-q),
$$

and, consequently,

$$
\mathbb{P}\left(|\eta| \leqslant \frac{1}{16} \varepsilon n|A|\right)=q \leqslant \frac{1-\varepsilon / 6}{1-\varepsilon / 12} \leqslant 1-\frac{1}{12} \varepsilon .
$$

First consider the case in which $m>2$. Putting together (8)-(10) and (16) we see that there are sets $X$ and $Y \subset \Gamma$ for which we have

$$
\begin{aligned}
|X| & =\left(\frac{1}{2}+o(1)\right) n, & & |Y|=\left(\frac{1}{2}+o(1)\right) n \\
|X \cap Y| & =\left(\frac{3}{8}+o(1)\right) n, & & |X \cup Y|=\left(\frac{5}{8}+o(1)\right) n,
\end{aligned}
$$

and

$$
\left|e(X, Y)-\frac{1}{4} n\right| A|| \geqslant \frac{1}{16} \varepsilon n|A| .
$$

\footnotetext{
${ }^{2}$ In (13), we write $(-X)$ for the characteristic function of the set $-X=\{-x: x \in X\}$.
} 
Fix such sets $X$ and $Y$. Suppose that none of the sets $X \backslash Y, Y \backslash X, X \cup Y$, and $X \cap Y$ violates $\operatorname{DISC}(\delta)$. Then for sufficiently large $n$ we have

$$
\left|e(X \backslash Y)-\frac{1}{128} n\right| A||<\frac{2}{128} \delta n|A|, \quad\left|e(Y \backslash X)-\frac{1}{128} n\right| A||<\frac{2}{128} \delta n|A|,
$$

and

$$
\left|e(X \cap Y)-\frac{9}{128} n\right| A||<\frac{10}{128} \delta n|A|, \quad\left|e(Y \cup X)-\frac{25}{128} n\right| A||<\frac{26}{128} \delta n|A| .
$$

Since

$$
e(X, Y)=e(X \cup Y)-e(X \backslash Y)-e(Y \backslash X)+e(X \cup Y),
$$

we infer that

$$
\left|e(X, Y)-\frac{32}{128} n\right| A||<\frac{40}{128} \delta n|A|
$$

which contradicts (17) if $\delta \leqslant \varepsilon / 5$. The proof for the case $m>2$ is finished.

The case $m=2$ is similar. Putting together (8), (9), (11), (12), and (16) we see that there are sets $X$ and $Y \subset \Gamma$ for which we have

$$
\begin{array}{cc}
|X|=\left(\frac{1}{2}+o(1)\right) n, \quad|Y|=\left(\frac{1}{2}+o(1)\right) n, \\
|X \cap Y|=\left(\frac{1}{2}+o(1)\right) n, \quad|X \cup Y|=\left(\frac{1}{2}+o(1)\right) n,
\end{array}
$$

and, moreover, with $X$ and $Y$ satisfying (17). Fix such sets $X$ and $Y$. Note that, then,

$$
e(X \backslash Y)=o(n|A|) \quad \text { and } \quad e(Y \backslash X)=o(n|A|) .
$$

Suppose that neither $X \cup Y$ nor $X \cap Y$ violates $\operatorname{DISC}(\delta)$. Then for sufficiently large $n$ we have

$$
\left|e(X \cap Y)-\frac{1}{8} n\right| A||<\frac{2}{8} \delta n|A| \quad \text { and } \quad\left|e(Y \cup X)-\frac{1}{8} n\right| A||<\frac{2}{8} \delta n|A| .
$$

Using (18) again, we infer that

$$
\left|e(X, Y)-\frac{1}{4} n\right| A||<\frac{5}{8} \delta n|A|,
$$

which contradicts (17) if $\delta \leqslant \varepsilon / 10$, completing the proof in the case $m=2$. 
2.3. Proof of Lemma 2.3. We start with the following fact (Fact $2.4(i)$ below is simply Lemma $2.3(i))$.

Fact 2.4. We have

$$
\sum_{\gamma \in \Gamma} c^{2}(\gamma)= \begin{cases}n & \text { if } m=2 \\ n / 2 & \text { if } m>2\end{cases}
$$

(ii)

$$
\sum_{\gamma \in \Gamma} s(\gamma) c(\gamma)=0
$$

(iii) for any $a \in \Gamma$

$$
(c * c)(a)= \begin{cases}n c(a) & \text { if } m=2 \\ (n / 2) c(a) & \text { if } m>2\end{cases}
$$

Proof. (i) We start by observing that

$$
\sum_{0 \leqslant \ell<m} \cos \frac{4 \pi \ell}{m}= \begin{cases}2 & \text { if } m=2 \\ 0 & \text { if } m>2\end{cases}
$$

Indeed, if $m>2$, then the sum in (22) is

$$
\operatorname{Re} \sum_{0 \leqslant \ell<m} \mathrm{e}^{4 \pi \ell \mathrm{i} / m}=\operatorname{Re} \frac{1-\mathrm{e}^{4 \pi \mathrm{i}}}{1-\mathrm{e}^{4 \pi \mathrm{i} / m}}=0 .
$$

If $m=2$, then the sum in (22) is easily seen to be 2 . We now observe that

$$
\sum_{\gamma \in \Gamma} c^{2}(\gamma)=\frac{n}{m} \sum_{0 \leqslant \ell<m} \cos ^{2}\left(\frac{2 \pi \ell}{m}\right)=\frac{n}{2 m} \sum_{0 \leqslant \ell<m}\left(1+\cos \frac{4 \pi \ell}{m}\right) .
$$

It now suffices to recall (22) to deduce (19); assertion $(i)$ is therefore proved.

Now we prove $(i i)$. Note that

$$
\sum_{0 \leqslant \ell<m} \sin \frac{4 \pi \ell}{m}=0
$$

Therefore,

$$
\sum_{\gamma \in \Gamma} s(\gamma) c(\gamma)=\frac{n}{m} \sum_{0 \leqslant \ell<m} \sin \left(\frac{2 \pi \ell}{m}\right) \cos \left(\frac{2 \pi \ell}{m}\right)=\frac{n}{2 m} \sum_{0 \leqslant \ell<m} \sin \frac{4 \pi \ell}{m}=0
$$

as required. 
For the proof of $(i i i)$, we start by noticing that

$$
\begin{aligned}
c(a-\gamma) & =\cos \left(\chi_{\mathrm{ARG}}(a-\gamma)\right)=\cos \left(\chi_{\mathrm{ARG}}(a)-\chi_{\mathrm{ARG}}(\gamma)\right) \\
& =\cos \chi_{\mathrm{ARG}}(a) \cos \chi_{\mathrm{ARG}}(\gamma)+\sin \chi_{\mathrm{ARG}}(a) \sin \chi_{\mathrm{ARG}}(\gamma)=c(a) c(\gamma)+s(a) s(\gamma)
\end{aligned}
$$

Therefore,

$$
\begin{aligned}
(c * c)(a) & =\sum_{\gamma \in \Gamma} c(a-\gamma) c(\gamma)=\sum_{\gamma \in \Gamma}(c(a) c(\gamma)+s(a) s(\gamma)) c(\gamma) \\
& =\sum_{\gamma \in \Gamma}\left(c(a) c^{2}(\gamma)+s(a) s(\gamma) c(\gamma)\right)=c(a) \sum_{\gamma \in \Gamma} c^{2}(\gamma)+s(a) \sum_{\gamma \in \Gamma} s(\gamma) c(\gamma)
\end{aligned}
$$

Eq. (21) follows from (19) and (20) and ( $i i i)$ is proved.

Proof of Lemma 2.3. Lemma $2.3(i)$ has already been proved. We now turn to $(i i)$. The left-hand side of (6) is

$$
\begin{aligned}
\frac{1}{4} \sum_{a \in A} \sum_{\gamma \in \Gamma}((1+c) & (a-\gamma))((1+c)(\gamma)) \\
= & \frac{1}{4} \sum_{a \in A} \sum_{\gamma \in \Gamma}(1+c(a-\gamma))(1+c(\gamma)) \\
= & \frac{1}{4} n|A|+\frac{1}{4} \sum_{a \in A} \sum_{\gamma \in \Gamma}(c(a-\gamma)+c(\gamma))+\frac{1}{4} \sum_{a \in A} \sum_{\gamma \in \Gamma} c(a-\gamma) c(\gamma) \\
= & \frac{1}{4} n|A|+\frac{1}{4} \sum_{a \in A} \sum_{\gamma \in \Gamma} c(a-\gamma) c(\gamma) \\
= & \frac{1}{4} n|A|+\frac{1}{4}\langle A, c * c\rangle
\end{aligned}
$$

which verifies (6). Clearly, Fact 2.4 (iii) and (23) imply (7).

\section{Appendix A}

A.1. Combinatorial proof of Theorem 1.6. We include our original proof of Theorem 1.6 here. Let a constant $\varepsilon>0$ be given. The aim is to find some $\delta>0$ for which property $\operatorname{DISC}(\delta)$ implies $\operatorname{EIG}(\varepsilon)$ for any Cayley graph $G=G(\Gamma, A)$. Let us once and for all fix an abelian group $\Gamma$ and a symmetric set $A \subseteq \Gamma \backslash\{0\}$. In what follows, we write $G$ for the Cayley graph $G(\Gamma, A)$. We shall always write $n$ for the number of vertices in $G$, i.e., $n=|\Gamma|=|V(G)|$. We also let $|A|=\alpha n$.

Clearly, our graph $G$ is $|A|$-regular. Therefore, the density of the graph $G$ is

$$
e(G) /\left(\begin{array}{l}
n \\
2
\end{array}\right)=\frac{|A|}{n-1} .
$$


Moreover, as is well known, condition $(i)$ of Definition 1.4 is automatically fulfilled. We should therefore consider condition $(i i)$ of that definition. Because of Theorem 2.1, our task is to estimate the $\lambda^{(\chi)}$ given in (3). More precisely, we have to show that if $\chi \neq \equiv 1$, then

$$
\left|\lambda^{(\chi)}\right|=\left|\sum_{a \in A} \chi(a)\right| \leqslant \varepsilon|A| .
$$

Thus, let $\chi: \Gamma \rightarrow S^{1}$ be a fixed, non-constant irreducible character of $\Gamma$. We shall estimate $\lambda^{(\chi)}$ in two different ways, according to the cardinality of $\operatorname{im} \chi=\{\chi(\gamma): \gamma \in \Gamma\}$. In what follows, we always write $m$ for $|\operatorname{im} \chi|$. We also use the bijection $\mathrm{e}^{\vartheta \mathbf{i}}$, mapping every $\vartheta$ in $\mathbb{R} / 2 \pi \mathbb{R}$ to $\mathrm{e}^{\vartheta \mathbf{i}}$ in $S^{1}$. We define

$$
\chi_{\mathrm{ARG}}: \Gamma \rightarrow \mathbb{R} / 2 \pi \mathbb{R}
$$

to be the homomorphism such that for every $\gamma \in \Gamma$

$$
\chi_{\mathrm{ARG}}(\gamma)=\arg (\chi(\gamma)) \quad \text { and } \quad \chi(\gamma)=\mathrm{e}^{\chi_{\mathrm{ARG}}(\gamma) \mathbf{i}}
$$

Furthermore, we let $\Omega: \mathbb{Z} / m \mathbb{Z} \rightarrow \mathbb{R} / 2 \pi \mathbb{R}$ be the homomorphism

$$
\Omega(s)=\frac{2 \pi}{m} s \quad \text { for } \quad s \in \mathbb{Z} / m \mathbb{Z} .
$$

We also have a homomorphism $\varrho: \Gamma \rightarrow \mathbb{Z} / m \mathbb{Z}$ for which $\chi_{\mathrm{ARG}}=\Omega \varrho$ holds, so that

$$
\chi_{\mathrm{ARG}}(\gamma)=\frac{2 \pi \varrho(\gamma)}{m}
$$

for every $\gamma$ in $\Gamma$. Summarising the above, from now on we will work with the following setup.

Setup A.5. Let $G=G(\Gamma, A)$ be the Cayley graph given by the abelian group $\Gamma$ and the symmetric set $A=-A \subseteq \Gamma \backslash\{0\}$. The graph $G$ is of order $n=|\Gamma|$, every vertex has degree $|A|=\alpha n$, and the density of the graph is $|A| /(n-1)$.

Fix an irreducible character $\chi \neq \equiv 1$, set $m=|\operatorname{im} \chi|$, and let $\chi_{\mathrm{ARG}}, \Omega$, and $\varrho$ (depending on $\chi$ ) be group homomorphisms such that the following diagram commutes:

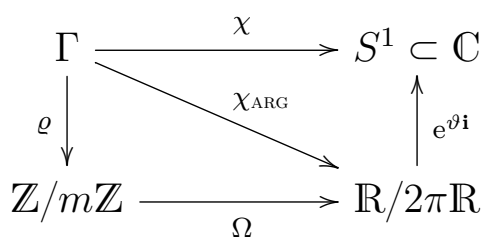

As mentioned above we consider two cases for the proof of Theorem 1.6. In the first case $m$ will be small. The following lemma will handle that case. 
Lemma A.6. For every $\delta^{\prime}>0$ there is an $n_{0} \geqslant 0$ such that if $|\Gamma|=n \geqslant n_{0}, m \leqslant 1 / \delta^{\prime}$, and $G=G(\Gamma, A)$ satisfies $\operatorname{DISC}_{2}\left(\delta^{\prime}\right)$, then

$$
\left|\lambda^{(\chi)}\right| \leqslant 2 \delta^{\prime}|A| .
$$

For the other case ( $m$ large), we shall need three auxiliary lemmas to verify (24). The proofs of these three lemmas, as well as the proof of Lemma A.6, are given in Sections A.2.2A.2.4. We start with two definitions.

Definition A.7 ( $\mathbb{Z}$-INT-DISC $(\varrho ; \eta, \sigma))$. For positive reals $\eta$ and $\sigma$, we say that $A$ satisfies $\mathbb{Z}$-INT-DISC $(\varrho ; \eta, \sigma)$ if for all integers $0 \leqslant D_{1}<D_{2} \leqslant\lfloor m / 2\rfloor+1$ such that $D_{2}-D_{1} \geqslant \eta m$ we have

$$
\left|A \cap \varrho^{-1}\left(\left[D_{1}, D_{2}\right)\right)\right| \sim_{\sigma} \frac{D_{2}-D_{1}}{m}|A| .
$$

Roughly speaking, a set $A$ satisfies $\mathbb{Z}$-INT-DISC if its image under $\varrho$ intersects "large" intervals uniformly. Next we define a very similar property for $A$ with respect to $\chi_{\mathrm{ARG}}$ and intervals in $\mathbb{R} / 2 \pi \mathbb{R}$.

Definition A.8 $\left(S^{1}\right.$-ARC-DISC $\left.\left(\chi_{\mathrm{ARG}} ; \eta, \sigma\right)\right)$. For positive reals $\eta$ and $\sigma$, we say that $A$ satisfies $S^{1}$-ARC-DISC $\left(\chi_{\mathrm{ARG}} ; \eta, \sigma\right)$ if for all reals $0 \leqslant \vartheta_{1}<\vartheta_{2} \leqslant \pi$ such that $\vartheta_{2}-\vartheta_{1} \geqslant 2 \pi \eta$ we have

$$
\left|A \cap \chi_{\mathrm{ARG}}^{-1}\left(\left[\vartheta_{1}, \vartheta_{2}\right]\right)\right| \sim_{\sigma} \frac{\vartheta_{2}-\vartheta_{1}}{2 \pi}|A| .
$$

Basically, the next three lemmas give the following implications for large $m$ :

$$
\text { DISC } \Longrightarrow \mathbb{Z} \text {-INT-DISC } \Longrightarrow S^{1} \text {-ARC-DISC } \Longrightarrow \text { EIG . }
$$

These lemmas are stated under the assumptions of Setup A.5; in particular, we recall that $\varrho$ and $\chi_{\mathrm{ARG}}$ depend on the fixed, non-constant character $\chi$.

Lemma A.9. For all positive reals $\eta$ and $\sigma$, there are $\delta=\delta(\eta, \sigma)>0$ and $n_{0} \geqslant 0$ such that if $|\Gamma|=n \geqslant n_{0}, m>1 / \delta$, and $G=G(\Gamma, A)$ satisfies $\operatorname{DISC}(\delta)$, then $A$ satisfies $\mathbb{Z}$-INT-DISC $(\varrho ; \eta, \sigma)$.

Lemma A.10. For all positive reals $\eta \leqslant 1$ and $\sigma \leqslant 1$ such that $m \eta \sigma \geqslant 3$, the following holds. If $A$ satisfies property $\mathbb{Z}$-INT-DISC $(\varrho ; \eta / 2, \sigma / 3)$, then A satisfies property $S^{1}$-ARC-DISC $\left(\chi_{\mathrm{ARG}} ; \eta, \sigma\right)$.

Lemma A.11. For every real $\varepsilon>0$, there are reals $\eta=\eta(\varepsilon)>0$ and $\sigma=\sigma(\varepsilon)>0$ for which the following holds. If A satisfies $S^{1}$-ARC-DISC $\left(\chi_{\mathrm{ARG}} ; \eta, \sigma\right)$, then

$$
\left|\lambda^{(\chi)}\right|=\left|\sum_{a \in A} \chi(a)\right| \leqslant \varepsilon|A| .
$$


We now assume Lemmas A.6, A.9, A.10, and A.11 and give the proof of Theorem 1.6. (We present the proofs of those auxiliary results in Section A.2.)

Proof of Theorem 1.6. Let $\varepsilon>0$ be given. We apply Lemma A.11, which yields the positive constants $\eta=\eta(\varepsilon)$ and $\sigma=\sigma(\varepsilon)$. Then Lemma A.9 gives $\delta_{A .9}=\delta_{A .9}(\eta / 2, \sigma / 3)$. We set

$$
\delta^{\prime}=\min \left\{\delta_{A .9}, \frac{\eta \sigma}{3}, \frac{\varepsilon}{2}\right\} .
$$

We now choose $\delta$ promised by Theorem 1.6 to be

$$
\delta=\min \left\{\delta_{1.3}\left(\delta^{\prime}\right), \delta_{A .9}\right\},
$$

where $\delta_{1.3}\left(\delta^{\prime}\right)$ is given by Fact 1.3. Finally, let $n_{0}$ be as large as required by Lemmas A.6 and A.9. We claim that this choice for $\delta$ and $n_{0}$ will do, and proceed to check this claim.

Suppose $\operatorname{DISC}(\delta)$ holds for some Cayley graph $G=G(\Gamma, A)$ with $|\Gamma| \geqslant n_{0}$ and let $\chi \not \equiv 1$ be given (the notation here follows the notation set out in Setup A.5). We consider two cases.

Suppose first that $m \leqslant 1 / \delta^{\prime}$. Fact 1.3 tells us that $\operatorname{DISC}_{2}\left(\delta^{\prime}\right)$ holds for $G$. Since $m \leqslant 1 / \delta^{\prime}$, Lemma A.6 tells us that $\left|\lambda^{(\chi)}\right| \leqslant \varepsilon|A|$ by the choice of $\delta^{\prime} \leqslant \varepsilon / 2$. For the other case, namely, $m>1 / \delta^{\prime}$, we first observe that $\operatorname{DISC}\left(\delta_{A .9}\right)$ holds since $\delta \leqslant \delta_{A .9}$ and that $m>1 / \delta^{\prime} \geqslant 1 / \delta_{A .9}$. Moreover, the choice of $\delta^{\prime} \leqslant \eta \sigma / 3$ yields $m \eta \sigma>\eta \sigma / \delta^{\prime} \geqslant 3$, making Lemma A.10 applicable. Our claim is now a consequence of the following implications coming from Lemmas A.9A.11:

$$
\operatorname{DISC}\left(\delta_{A .9}\right) \Longrightarrow \mathbb{Z} \text {-INT-DISC }\left(\varrho ; \frac{\eta}{2}, \frac{\sigma}{3}\right) \Longrightarrow S^{1} \text {-ARC-DISC }\left(\chi_{\mathrm{ARG}} ; \eta, \sigma\right) \Longrightarrow\left|\lambda^{(\chi)}\right| \leqslant \varepsilon|A|,
$$

and hence Theorem 1.6 is proved.

A.2. Proof of the auxiliary lemmas. In this section we prove the auxiliary lemmas Lemmas A.6, A.9, A.10, and A.11

A.2.1. An auxiliary weighted graph. The homomorphism $\varrho$ (see Setup A.5 for details), defines a weighted graph $\widetilde{G}$ on $\mathbb{Z} / m \mathbb{Z}$ in a natural way. The symmetry of this graph will be useful in the proofs of Lemmas A.6 and A.9.

Definition A.12. We let (under the assumptions of Setup A.5) $\widetilde{G}=\widetilde{G}(\varrho)=(\mathbb{Z} / m \mathbb{Z}, w)$ be the weighted graph on $\mathbb{Z} / m \mathbb{Z}$, with weights assigned to the edges and vertices, with the weight function

$$
w:\left(\begin{array}{c}
\mathbb{Z} / m \mathbb{Z} \\
2
\end{array}\right) \cup \mathbb{Z} / m \mathbb{Z} \rightarrow \mathbb{Z}
$$

given by

$$
w(\{r, s\})=e\left(G\left[\varrho^{-1}(r), \varrho^{-1}(s)\right]\right),
$$


for all distinct $r$ and $s \in \mathbb{Z} / m \mathbb{Z}$, and

$$
w(r)=e\left(G\left[\varrho^{-1}(r)\right]\right)
$$

for all $r \in \mathbb{Z} / m \mathbb{Z}$.

For convenience, if $X$ and $Y \subset \mathbb{Z} / m \mathbb{Z}$ are two disjoint sets, we put

$$
w(X, Y)=\sum\{w(\{x, y\}):(x, y) \in X \times Y\}=e\left(\varrho^{-1}(X), \varrho^{-1}(Y)\right) .
$$

In Lemma A.13 below, we make the definition of $\widetilde{G}$ more concrete, computing the values in (27) and (28). Let us observe that Lemma A.13 shows that the weighted graph $\widetilde{G}$ has a "cyclic" structure, that is, the cyclic permutation $\tau: s \mapsto s+1$ is an "automorphism" of $\widetilde{G}$.

Lemma A.13. For all distinct $r$ and $s \in \mathbb{Z} / m \mathbb{Z}$, we have

$$
w(\{r, s\})=e\left(G\left[\varrho^{-1}(r), \varrho^{-1}(s)\right]\right)=\frac{n}{m}\left|A \cap \varrho^{-1}(r-s)\right|,
$$

and for all $r \in \mathbb{Z} / m \mathbb{Z}$ we have

$$
w(r)=e\left(G\left[\varrho^{-1}(r)\right]\right)=\frac{n}{2 m}\left|A \cap \varrho^{-1}(0)\right| .
$$

Proof. Let $r$ and $s$ be arbitrary, not necessarily distinct members of $\mathbb{Z} / m \mathbb{Z}$. For every $\gamma$ in $\varrho^{-1}(s)$, consider the neighbourhood $N_{r}(\gamma)$ of $\gamma$ in $G$ restricted to $\varrho^{-1}(r)$. It is easy to see that

$$
N_{r}(\gamma)=\left\{A \cap \varrho^{-1}(r-s)\right\}+\gamma
$$

for every $\gamma \in \varrho^{-1}(s)$. Since $\left|\varrho^{-1}(s)\right|=n / m$, this implies, for $s \neq r$, that

$$
e\left(G\left[\varrho^{-1}(r), \varrho^{-1}(s)\right]\right)=\left|\varrho^{-1}(s)\right| \cdot\left|A \cap \varrho^{-1}(r-s)\right|=\frac{n}{m}\left|A \cap \varrho^{-1}(r-s)\right|,
$$

and therefore (29) holds. Similarly, (30) follows from the case $r=s$.

A.2.2. The small $m$ case. The proof given in this section is fairly simple. It is based on (29) combined with an application of $\mathrm{DISC}_{2}$.

Proof of Lemma A.6. Let $\delta^{\prime}>0$ be given and let $n_{0}$ large enough such that $n \sim_{\delta^{\prime} / 2}(n-1)$ for every $n \geqslant n_{0}$.

Now assume $G=G(\Gamma, A)$ with $|\Gamma| \geqslant n_{0}$ satisfying $\operatorname{DISC}_{2}\left(\delta^{\prime}\right)$ is given. Using $1 / m \geqslant \delta^{\prime}$, we deduce from $\operatorname{DISC}_{2}\left(\delta^{\prime}\right)$ and (29) that for all $r \in \mathbb{Z} / m \mathbb{Z}$

$$
\left|A \cap \varrho^{-1}(r)\right|=\frac{m}{n} e\left(G\left[\varrho^{-1}(r), \varrho^{-1}(0)\right]\right) \sim_{\delta^{\prime}} \frac{n|A|}{(n-1) m},
$$

and hence, by the choice of $n_{0}$,

$$
\left|A \cap \varrho^{-1}(r)\right| \leqslant\left(1+2 \delta^{\prime}\right) \frac{|A|}{m} .
$$


We then set $\omega=\mathrm{e}^{2 \pi \mathrm{i} / m}$ and use (31) to infer

$$
\left|\lambda^{(\chi)}\right|=\left|\sum_{a \in A} \chi(a)\right|=\left|\sum_{r=0}^{m-1}\left(\left|A \cap \varrho^{-1}(r)\right| \cdot \omega^{r}\right)\right| \leqslant \frac{|A|}{m}\left(\left|\sum_{r=0}^{m-1} \omega^{r}\right|+2 \delta^{\prime} m\right),
$$

which yields $\left|\lambda^{(\chi)}\right| \leqslant 2 \delta^{\prime}|A|$, because

$$
\sum_{r=0}^{m-1} \omega^{r}=0
$$

A.2.3. DISC implies $\mathbb{Z}$-INT-DISC for $m$ large. The aim of this section is to verify $\mathbb{Z}$-INT-DISC $(\varrho ; \eta, \sigma)$ for a graph that satisfies $\operatorname{DISC}(\delta)$ for sufficiently small $\delta$. We therefore want to link properties of the edge-distribution of $G$ with the quantities

$$
\left|A \cap \varrho^{-1}(I)\right|=\sum_{f \in I}\left|A \cap \varrho^{-1}(f)\right|,
$$

where $I$ is a sufficiently large interval in $\mathbb{Z} / m \mathbb{Z}$. A first step towards this goal is the following lemma.

Lemma A.14. Let $\ell, s$, and $t$ be integers, and suppose that

$$
0 \leqslant s<s+\ell \leqslant t<t+\ell \leqslant m / 2 .
$$

Then for $d_{1}=t-s-\ell$ and $d_{2}=t-s+\ell$

$$
\begin{aligned}
\frac{m}{n} w([s, s+\ell),[t, t+\ell))=\sum & \left\{\left|A \cap \varrho^{-1}(f)\right|\left(f-d_{1}\right): d_{1}<f<t-s\right\} \\
& +\sum\left\{\left|A \cap \varrho^{-1}(f)\right|\left(d_{2}-f\right): t-s \leqslant f<d_{2}\right\} .
\end{aligned}
$$

We later may control the left-hand side of (32) by DISC (or, more precisely, by $\mathrm{DISC}_{2}$ ). On the other hand, we may interpret the right-hand side as a "weighted version" of $\left|A \cap \varrho^{-1}\left(\left[d_{1}, d_{2}\right]\right)\right|$ where the "multiplicity" for each $f$ in $\left[d_{1}, d_{2}\right]$ is given by a piecewise linear function depending on $d_{1}$ and $d_{2}$ (see Figure 1).

Proof of Lemma A.14. We have

$$
w([s, s+\ell),[t, t+\ell))=\sum\left\{w(e): e \in E_{\widetilde{G}}([s, s+\ell),[t, t+\ell))\right\} .
$$

The integers $f$ that arise as differences $t^{\prime}-s^{\prime}$ with $t^{\prime} \in[t, t+\ell)$ and $s^{\prime} \in[s, s+\ell)$ are in the interval

$$
d_{1}=t-s-\ell<f<t-s+\ell=d_{2} .
$$

Intuitively speaking, these are the "lengths" of the edges in $E_{\widetilde{G}}([s, s+\ell),[t, t+\ell))$. A moment's thought (see Figure 2) shows that assertions (I) and (II) given below hold. 


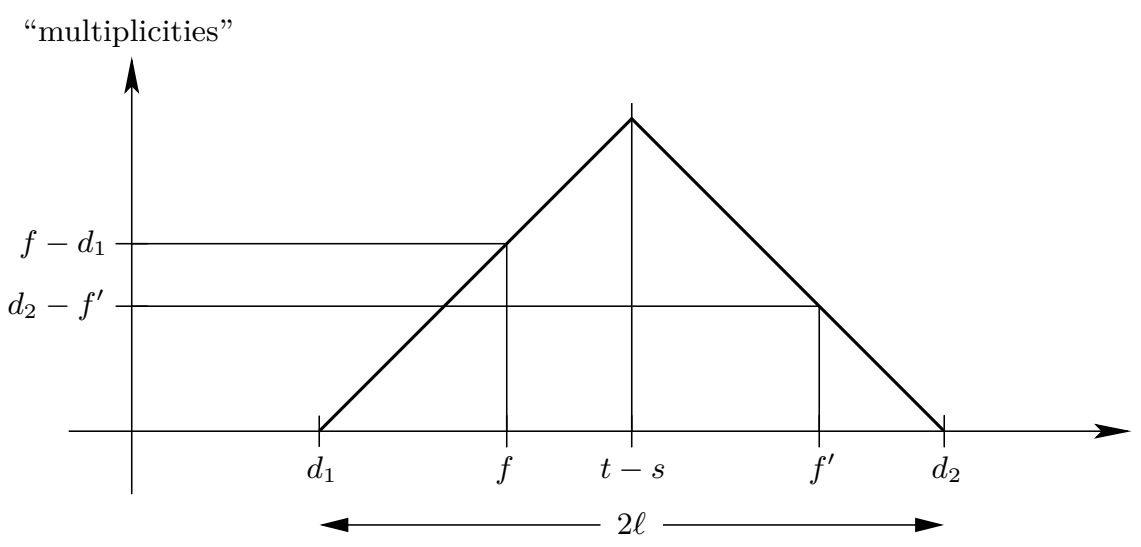

Figure 1. Distribution of "multiplicities"

(I) If $f$ is in the interval $d_{1}=t-s-\ell<f<t-s$, then $f-d_{1}$ edges in the graph $E_{\widetilde{G}}([s, s+\ell),[t, t+\ell))$ have length $f$.

(II) If $f$ is in the interval $t-s \leqslant f<d_{2}=t-s+\ell$, then $d_{2}-f$ edges in the graph $E_{\widetilde{G}}([s, s+\ell),[t, t+\ell))$ have length $f$.

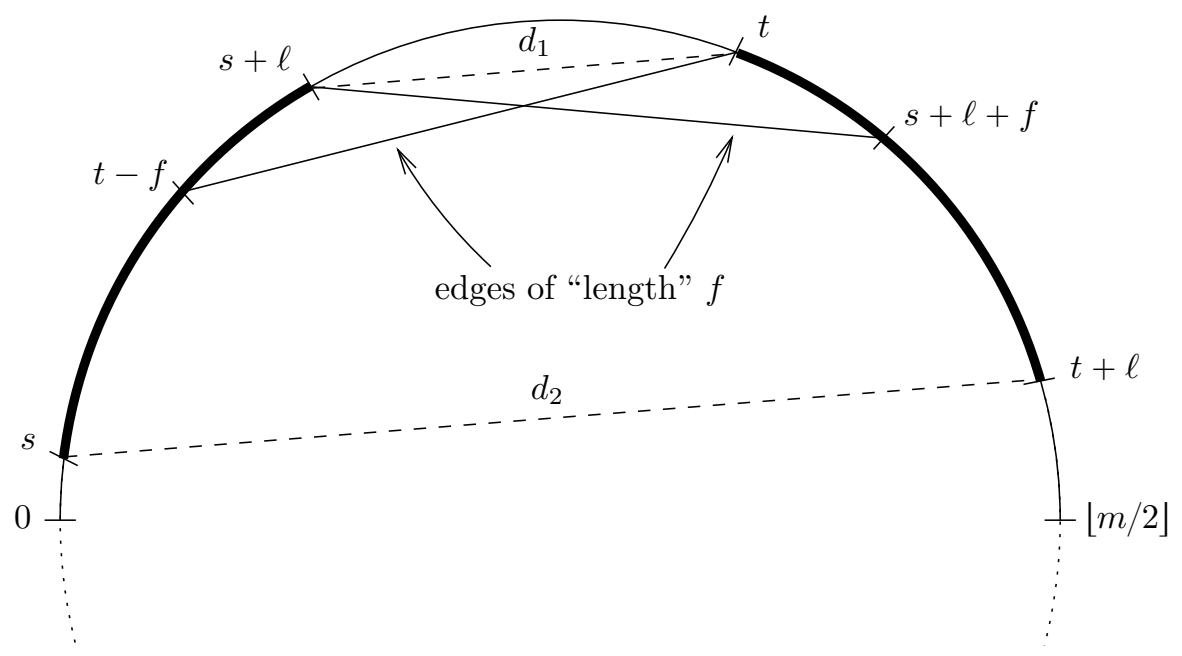

Figure 2. Edges in $\widetilde{G}$ of "length" $d_{1}<f<t-s$ appear between $[t-f, s+\ell)$ and $[t, s+\ell+f)$

In other words, the lengths $f$ in the interval $\left(d_{1}, t-s\right)$ occur $f-d_{1}$, times and the lengths $f$ in the interval $\left[t-s, d_{2}\right.$ ) occur $d_{2}-f$ times in the sum in (33). Each occurrence of $f$ contributes to (33) a weight of

$$
\frac{n}{m}\left|A \cap \varrho^{-1}(f)\right|
$$

(see (29)). Therefore, putting (I), (II), and (34) together, identity (32) follows. 
The next step towards verifying $\mathbb{Z}$-INT-DISC is to dispose of the "multiplicities" of type $d_{1}-f$ and $d_{2}-f$ in (32). For this we use Lemma A.14 and we compare the quantities $w([s-d, s+\ell),[t-d, t+\ell))$ with $w([s, s+\ell),[t, t+\ell))$. By (32) these two terms (appropriately scaled) correspond to two "weighted versions" of $\left|A \cap \varrho^{-1}\left(\left[d_{1}^{\prime}, d_{2}^{\prime}\right]\right)\right|$ and $\left|A \cap \varrho^{-1}\left(\left[d_{1}, d_{2}\right]\right)\right|$, for some appropriate $d_{1}^{\prime}, d_{2}^{\prime}, d_{1}$, and $d_{2}$ depending on $d, s, t$, and $\ell$. As it turns out, the difference between these two "weighted versions" yields a "weighted version" of $\left|A \cap \varrho^{-1}\left(\left[d_{1}^{\prime}, d_{2}^{\prime}\right]\right)\right|$ with constant multiplicity $d$ for the main part of the interval $\left[d_{1}^{\prime}, d_{2}^{\prime}\right]$, i.e., in between $d_{1}$ and $d_{2}$ (see Figure 3 ). This way we derive a useful estimate for $\left|A \cap \varrho^{-1}\left(\left[d_{1}, d_{2}\right]\right)\right|$.

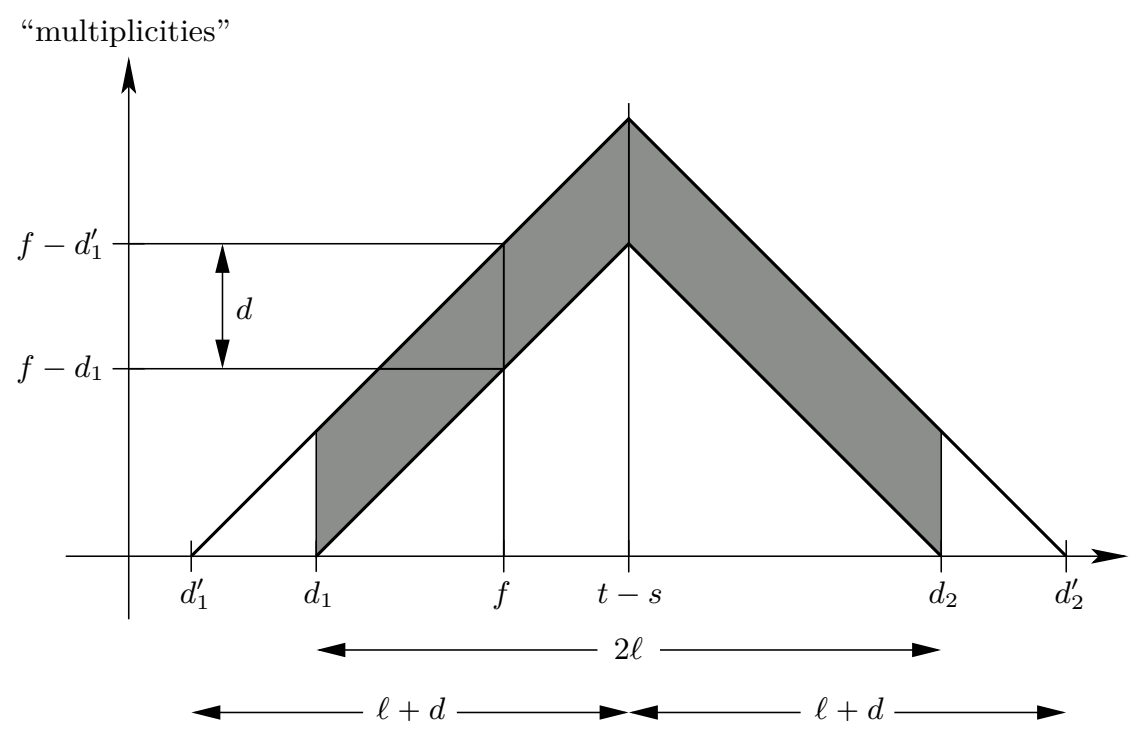

FiguRE 3. Difference between "multiplicities"

Lemma A.15. Let $d, \ell, s$, and $t$ be positive integers, $\delta^{\prime}$ a real number such that $0<\delta^{\prime} \leqslant 1$, and suppose that

(i) $0 \leqslant s-d<s+\ell \leqslant t-d<t+\ell \leqslant m / 2$,

(ii) $\mathrm{DISC}_{2}\left(\delta^{\prime}\right)$ holds for $G=G(\Gamma, A)$,

(iii) $\ell-d \geqslant \delta^{\prime} m$, and

(iv) $n \sim_{\delta^{\prime} / 2}(n-1)$.

Then, for $d_{1}=t-s-\ell$ and $d_{2}=t-s+\ell$, we have

$$
|| A \cap \varrho^{-1}\left(\left[d_{1}, d_{2}\right)\right)\left|-2 \ell \frac{|A|}{m}\right| \leqslant \frac{|A|}{m}\left(d+\frac{2 \delta^{\prime}}{d}\left((\ell+d)^{2}+\ell^{2}\right)\right) .
$$


Proof. Let $d_{1}^{\prime}=(t-d)-s-\ell=d_{1}-d$ and $d_{2}^{\prime}=t+\ell-(s-d)=d_{2}+d$. Applying Lemma A.14 to $w([s-d, s+\ell),[t-d, t+\ell))$, we get that

$$
\begin{aligned}
\frac{m}{n} w([s-d, s+\ell),[ & -d, t+\ell)) \\
=\sum\left\{\left|A \cap \varrho^{-1}(f)\right|\right. & \left.\left(f-d_{1}^{\prime}\right): d_{1}^{\prime}<f<t-s\right\} \\
& +\sum\left\{\left|A \cap \varrho^{-1}(f)\right|\left(d_{2}^{\prime}-f\right): t-s \leqslant f<d_{2}^{\prime}\right\} .
\end{aligned}
$$

We then apply Lemma A.14 again, now to $w([s, s+\ell),[t, t+\ell))$, and observe that

$$
\begin{aligned}
& \frac{m}{n}(w([s-d, s+\ell),[t-d, t+\ell))-w([s, s+\ell),[t, t+\ell))) \\
&=d \sum\left\{\left|A \cap \varrho^{-1}(f)\right|: d_{1} \leqslant f<d_{2}\right\} \\
&+\sum\left\{\left|A \cap \varrho^{-1}(f)\right|\left(f-d_{1}^{\prime}\right): d_{1}^{\prime}<f<d_{1}\right\} \\
&+\sum\left\{\left|A \cap \varrho^{-1}(f)\right|\left(d_{2}^{\prime}-f\right): d_{2} \leqslant f<d_{2}^{\prime}\right\} .
\end{aligned}
$$

The "main term" on the right-hand side of (36) will turn out to be

$$
d \sum\left\{\left|A \cap \varrho^{-1}(f)\right|: d_{1} \leqslant f<d_{2}\right\}=d\left|A \cap \varrho^{-1}\left(\left[d_{1}, d_{2}\right)\right)\right| .
$$

We now use $\mathrm{DISC}_{2}\left(\delta^{\prime}\right)$ to estimate the left-hand side of (36). By the definition of $w$ (see Definition A.12), we have

$$
w([s, s+\ell),[t, t+\ell))=e\left(G\left[\varrho^{-1}([s, s+\ell)), \varrho^{-1}([t, t+\ell))\right]\right) .
$$

Therefore, by $\mathrm{DISC}_{2}\left(\delta^{\prime}\right)$, using that

$$
\left|\varrho^{-1}([s, s+\ell))\right|=\left|\varrho^{-1}([t, t+\ell))\right|=\frac{n}{m} \ell \geqslant \delta^{\prime} n,
$$

we have that

$$
\begin{aligned}
w([s, s+\ell),[t, t+\ell)) & =e\left(G\left[\varrho^{-1}([s, s+\ell)), \varrho^{-1}([t, t+\ell))\right]\right) \\
& \sim \sim_{\delta^{\prime}} \frac{|A|}{n-1}\left|\varrho^{-1}([s, s+\ell))\right|\left|\varrho^{-1}([t, t+\ell))\right|=\frac{|A|}{n-1}\left(\frac{n}{m} \ell\right)^{2} .
\end{aligned}
$$

Similarly, we have that

$$
w([s-d, s+\ell),[t-d, t+\ell)) \sim_{\delta^{\prime}} \frac{|A|}{n-1}\left(\frac{n}{m}(\ell+d)\right)^{2} .
$$

From (38), (39), and ( $i v)$ we deduce that the left-hand side of (36) satisfies

$$
\begin{gathered}
\frac{m}{n}(w([s-d, s+\ell),[t-d, t+\ell))-w([s, s+\ell),[t, t+\ell))) \\
=\left(1+O_{1}\left(2 \delta^{\prime}\right)\right) \frac{|A|}{m}(\ell+d)^{2}-\left(1+O_{1}\left(2 \delta^{\prime}\right)\right) \frac{|A|}{m} \ell^{2} \\
=\frac{|A|}{m}\left(2 \ell d+d^{2}+O_{1}\left(2 \delta^{\prime}\right)\left((\ell+d)^{2}+\ell^{2}\right)\right) .
\end{gathered}
$$


Therefore, replacing the left-hand side of (36) by (40) and using (37) immediately yields

$$
\begin{aligned}
d\left|A \cap \varrho^{-1}\left(\left[d_{1}, d_{2}\right)\right)\right| & \\
& +\sum\left\{\left|A \cap \varrho^{-1}(f)\right|\left(f-d_{1}^{\prime}\right): d_{1}^{\prime}<f<d_{1}\right\} \\
& +\sum\left\{\left|A \cap \varrho^{-1}(f)\right|\left(d_{2}^{\prime}-f\right): d_{2} \leqslant f<d_{2}^{\prime}\right\} \\
& =\frac{|A|}{m}\left(2 \ell d+d^{2}+O_{1}\left(2 \delta^{\prime}\right)\left((\ell+d)^{2}+\ell^{2}\right)\right) .
\end{aligned}
$$

Clearly, (41) implies that

$$
d\left|A \cap \varrho^{-1}\left(\left[d_{1}, d_{2}\right)\right)\right| \leqslant \frac{|A|}{m}\left(2 \ell d+d^{2}+O_{1}\left(2 \delta^{\prime}\right)\left((\ell+d)^{2}+\ell^{2}\right)\right) .
$$

Moreover, we observe that

$$
\begin{aligned}
d\left|A \cap \varrho^{-1}\left(\left[d_{1}, d_{2}\right)\right)\right| \geqslant & d\left|A \cap \varrho^{-1}\left(\left[d_{1}+d, d_{2}-d\right)\right)\right| \\
& +\sum\left\{\left|A \cap \varrho^{-1}(f)\right|\left(f-d_{1}\right): d_{1} \leqslant f<d_{1}+d\right\} \\
& +\sum\left\{\left|A \cap \varrho^{-1}(f)\right|\left(d_{2}-f\right): d_{2}-d \leqslant f<d_{2}\right\} \\
= & \frac{m}{n}(w([s, s+\ell),[t, t+\ell))-w([s+d, s+\ell),[t+d, t+\ell))),
\end{aligned}
$$

where the last identity follows from Lemma A.14 in the same way that equation (36) follows from that lemma. Then essentially the same calculations as in (40) give

$$
\begin{aligned}
\frac{m}{n}(w([s, s+\ell),[t, t+\ell))-w([s+d, s+\ell),[t+d, t+\ell))) & \\
= & \frac{|A|}{m}\left(2(\ell-d) d+d^{2}+O_{1}\left(2 \delta^{\prime}\right)\left(\ell^{2}+(\ell-d)^{2}\right)\right),
\end{aligned}
$$

and hence

$$
d\left|A \cap \varrho^{-1}\left(\left[d_{1}, d_{2}\right)\right)\right| \geqslant \frac{|A|}{m}\left(2 \ell d-d^{2}+O_{1}\left(2 \delta^{\prime}\right)\left(\ell^{2}+(\ell-d)^{2}\right)\right) .
$$

Inequality (35) follows from (42) and (43), and thus Lemma A.15 is proved.

We prove a simple corollary of Lemma A.15 that allows us to rewrite the conditions of Lemma A.15 in terms of $d_{1}$ and $d_{2}$. Moreover, the hypotheses of Lemma A.15 imply that $d_{2}-d_{1}=2 \ell$ is even. The following corollary overcomes this shortcoming. 
Corollary A.16. Let $d, d_{1}$, and $d_{2}$ be positive integers, $\delta^{\prime}$ a real number such that $0<$ $\delta^{\prime} \leqslant 1$, and suppose that

(i) $0<d \leqslant d_{1}<d_{2}-1<d_{2}+1 \leqslant \frac{1}{2} m-d$,

(ii) $\operatorname{DISC}_{2}\left(\delta^{\prime}\right)$ holds for $G=G(\Gamma, A)$,

(iii) $\frac{1}{2}\left(d_{2}-d_{1}-1\right)-d \geqslant \delta^{\prime} m$,

(iv) $n \sim_{\delta^{\prime} / 2}(n-1)$.

Then

$$
|| A \cap \varrho^{-1}\left(\left[d_{1}, d_{2}\right)\right)\left|-\frac{d_{2}-d_{1}}{m}\right| A|| \leqslant \frac{|A|}{m}\left(d+1+\frac{\delta^{\prime}}{d} \cdot \frac{m^{2}}{4}\right) .
$$

Proof. We distinguish two cases depending on the parity of $d_{2}-d_{1}$. We later reduce the second case $\left(d_{2}-d_{1}\right.$ odd $)$ to the first case $\left(d_{2}-d_{1}\right.$ even). In order to be prepared for this we are going to show a stronger statement for the first case.

Case $1\left(d_{2}-d_{1}\right.$ is even). Let us consider the following weaker conditions $\left(i^{\prime}\right)$ and $\left(i i i^{\prime}\right)$ instead of $(i)$ and $(i i i)$ :

$$
\begin{gathered}
\left(i^{\prime}\right) \quad 0<d \leqslant d_{1}<d_{2} \leqslant \frac{m}{2}-d, \\
\left(i i i^{\prime}\right) \quad \frac{1}{2}\left(d_{2}-d_{1}\right)-d \geqslant \delta^{\prime} m .
\end{gathered}
$$

We are now going to show a stronger conclusion than (44) under these weaker assumptions. In particular, we shall verify

$$
|| A \cap \varrho^{-1}\left(\left[d_{1}, d_{2}\right)\right)\left|-\frac{d_{2}-d_{1}}{m}\right| A|| \leqslant \frac{|A|}{m}\left(d+\frac{\delta^{\prime}}{d} \cdot \frac{m^{2}}{4}\right) .
$$

For this we want to apply Lemma A.15 for the "right" choice of $s$, $t$, and $\ell$. First, note that $(i i)$ and $(i v)$ are the same in Lemma A.15 and Corollary A.16. We set

$$
s=d, \quad \ell=\frac{1}{2}\left(d_{2}-d_{1}\right), \quad \text { and } \quad t=s+\ell+d_{1} .
$$

Simple calculations using $\left(i^{\prime}\right)$ and $\left(i i i^{\prime}\right)$ show that

$$
0=s-d<s+\ell \leqslant t-d<t+\ell \leqslant \frac{1}{2} m \quad \text { and } \quad \ell-d \geqslant \delta^{\prime} m,
$$

and hence $(i)$ and $(i i i)$ of Lemma A.15 hold for this particular choice of $s, t$, and $\ell$. Moreover, $t-s-\ell=d_{1}$ and $t-s+\ell=d_{2}$, thus Lemma A.15 implies

$$
|| A \cap \varrho^{-1}\left(\left[d_{1}, d_{2}\right)\right)\left|-\frac{2 \ell}{m}\right| A|| \leqslant \frac{|A|}{m}\left(d+\frac{2 \delta^{\prime}}{d}\left((\ell+d)^{2}+\ell^{2}\right)\right),
$$


which, combined with

$$
\ell^{2} \leqslant(\ell+d)^{2}=\frac{\left(d_{2}-d_{1}+2 d\right)^{2}}{4}=\frac{\left(\left(d_{2}+d\right)+\left(d-d_{1}\right)\right)^{2}}{4} \leqslant \frac{(m / 2)^{2}}{4}=\frac{m^{2}}{16},
$$

gives inequality (45).

Case $2\left(d_{2}-d_{1}\right.$ is odd). The hypotheses of Lemma A.15 unfortunately always imply $\ell=d_{2}-d_{1}$ is even. In order to get a bound for intervals $\left[d_{1}, d_{2}\right)$ of odd length we "sandwich" $\left|A \cap \varrho^{-1}\left(\left[d_{1}, d_{2}\right)\right)\right|$ as follows:

$$
\left|A \cap \varrho^{-1}\left(\left[d_{1}, d_{2}-1\right)\right)\right| \leqslant\left|A \cap \varrho^{-1}\left(\left[d_{1}, d_{2}\right)\right)\right| \leqslant\left|A \cap \varrho^{-1}\left(\left[d_{1}, d_{2}+1\right)\right)\right| .
$$

Now we apply Case 1 twice to derive the upper and lower bounds in (44). For the upper bound, we set

$$
d_{2}^{\mathrm{U}}=d_{2}+1
$$

Then conditions $(i)$ and (iii) of Corollary A.16 are "strong" enough to imply $\left(i^{\prime}\right)$ and $\left(i i i^{\prime}\right)$ of Case 1, applied to $d_{2}^{\mathrm{U}}$ instead of $d_{2}$. Thus, by (45), we may bound the right-hand side of (46) from above by

$$
\begin{aligned}
\left|A \cap \varrho^{-1}\left(\left[d_{1}, d_{2}+1\right)\right)\right|=\mid A & \cap \varrho^{-1}\left(\left[d_{1}, d_{2}^{\mathrm{U}}\right)\right) \mid \\
\leqslant \frac{|A|}{m}\left(d+\frac{\delta^{\prime}}{d} \cdot \frac{m^{2}}{4}\right) & +\frac{d_{2}^{\mathrm{U}}-d_{1}}{m}|A| \\
& =\frac{|A|}{m}\left(d+1+\frac{\delta^{\prime}}{d} \cdot \frac{m^{2}}{4}\right)+\frac{d_{2}-d_{1}}{m}|A| .
\end{aligned}
$$

Hence, the upper bound for $\left|A \cap \varrho^{-1}\left(\left[d_{1}, d_{2}\right)\right)\right|$ in (44) follows. The lower bound necessary to complete the proof of Corollary A.16 may be verified by the same kind of argument applied to $d_{2}^{\mathrm{L}}=d_{2}-1$ instead of $d_{2}^{\mathrm{U}}$.

Corollary A.16 above gives us control over

$$
\left|A \cap \varrho^{-1}\left(\left[d_{1}, d_{2}\right)\right)\right|
$$

as long as $d_{1}$ and $d_{2}$ are bounded away from 0 and $m / 2$. The following two lemmas consider the quantities

$$
\left|A \cap \varrho^{-1}([0, d))\right| \text { and }\left|A \cap \varrho^{-1}\left(\left\lfloor\left\lfloor\frac{m}{2}\right\rfloor-d-1,\left\lfloor\frac{m}{2}\right\rfloor+1\right)\right)\right| .
$$

Lemma A.17. Suppose $0<\delta \leqslant 1 / 3, d \geqslant \delta m / 2$, and $n \geqslant 4$. If $\mathrm{DISC}(\delta)$ holds for the Cayley graph $G=G(\Gamma, A)$, then

$$
\left|A \cap \varrho^{-1}([0, d))\right| \leqslant 4 \frac{d}{m}|A| .
$$


Proof. Let $\delta>0$ and $d$ be as in the statement of the lemma, and assume that $G$ satisfies $\operatorname{DISC}(\delta)$. Let us estimate from above the number of edges induced by

$$
U=\varrho^{-1}([0,2 d))
$$

in $G$. We have $|U|=2 d n / m \geqslant \delta n$. Invoking $\operatorname{DISC}(\delta)$, using that $0<\delta \leqslant 1 / 3$ and $n \geqslant 4$, and recalling that $|A|=\alpha n$, we obtain that

$$
e(G[U]) \leqslant(1+\delta) \frac{|A|}{n-1}\left(\begin{array}{c}
|U| \\
2
\end{array}\right) \leqslant 4 \alpha\left(\frac{d n}{m}\right)^{2} .
$$

On the other hand, by Lemma A.13, we have

$$
e(G[U])=\sum_{r=0}^{2 d-1} \sum_{s=r+1}^{2 d-1} w(\{r, s\})+\sum_{r=0}^{2 d-1} w(r) \geqslant \sum_{r=0}^{d-1} \sum_{s=r+1}^{r+d-1} w(\{r, s\})+\sum_{r=0}^{2 d-1} w(r) .
$$

Now fix $0 \leqslant r<d$. If $r<s<r+d$, then $0<s-r<d$, and, by (29) of Lemma A.13, we have

$$
\begin{aligned}
\sum_{s=r+1}^{r+d-1} w(\{r, s\}) & =\frac{n}{m} \sum_{s=r+1}^{r+d-1}\left|A \cap \varrho^{-1}(s-r)\right| \\
& =\frac{n}{m} \sum_{f=1}^{d-1}\left|A \cap \varrho^{-1}(f)\right|=\frac{n}{m}\left|A \cap \varrho^{-1}([1, d))\right| .
\end{aligned}
$$

Also, by (30),

$$
w(r)=\frac{n}{2 m}\left|A \cap \varrho^{-1}(0)\right|,
$$

and we may conclude from (50) that

$$
e(G[U]) \geqslant d \frac{n}{m}\left|A \cap \varrho^{-1}([1, d))\right|+2 d \frac{n}{2 m}\left|A \cap \varrho^{-1}(0)\right|=d \frac{n}{m}\left|A \cap \varrho^{-1}([0, d))\right| .
$$

Comparing (49) and (51), inequality (48) follows and our lemma is proved.

Our next lemma concerns the second interval in (47).

Lemma A.18. Suppose $0<\delta^{\prime} \leqslant 1 / 3, d \geqslant \delta^{\prime} m$, and $n \geqslant 4$. If $\mathrm{DISC}_{2}\left(\delta^{\prime}\right)$ holds for the Cayley graph $G=G(\Gamma, A)$, then

$$
\left|A \cap \varrho^{-1}\left(\left[\left\lfloor\frac{m}{2}\right\rfloor-d-1,\left\lfloor\frac{m}{2}\right\rfloor+1\right)\right)\right| \leqslant 4 \frac{d+1}{m}|A| .
$$

Proof. Let $\delta^{\prime}>0$ and $d$ be as in the statement of the lemma, and assume that $G$ satisfies $\mathrm{DISC}_{2}\left(\delta^{\prime}\right)$. Let us estimate from above the number of edges in the bipartite graph induced by the vertex classes

$$
U=\varrho^{-1}([0, d)) \text { and } W=\varrho^{-1}([\lfloor m / 2\rfloor-d-1,\lfloor m / 2\rfloor+d))
$$


in $G$. We have $|U|=d n / m \geqslant \delta^{\prime} n$ and $|W|=(2 d+1) n / m>\delta^{\prime} n$. Invoking $\mathrm{DISC}_{2}\left(\delta^{\prime}\right)$ and using that $0<\delta^{\prime} \leqslant 1 / 3$ and $n \geqslant 4$, we obtain that

$$
e(G[U, W]) \leqslant\left(1+\delta^{\prime}\right) \frac{|A|}{n-1}|U||W| \leqslant 4 \alpha \frac{d(d+1) n^{2}}{m^{2}} .
$$

On the other hand, by Lemma A.13, we have

$$
\begin{aligned}
e(G[U, W]) & \geqslant \sum_{r=0}^{d-1} \sum\left\{w(\{r, s\}):\left\lfloor\frac{m}{2}\right\rfloor-d-1+r \leqslant s<\left\lfloor\frac{m}{2}\right\rfloor+1+r\right\} \\
& =\sum_{r=0}^{d-1} \frac{n}{m} \sum\left\{\left|A \cap \varrho^{-1}(f)\right|:\left\lfloor\frac{m}{2}\right\rfloor-d-1 \leqslant f<\left\lfloor\frac{m}{2}\right\rfloor+1\right\} \\
& =d \frac{n}{m}\left|A \cap \varrho^{-1}\left(\left\lfloor\left\lfloor\frac{m}{2}\right\rfloor-d-1,\left\lfloor\frac{m}{2}\right\rfloor+1\right)\right)\right| .
\end{aligned}
$$

Comparing (53) and (54), we deduce (52). Lemma A.18 is proved.

We may now prove Lemma A.9.

Proof of Lemma A.9. Let positive reals $\eta \leqslant 1$ and $\sigma \leqslant 1$ be given. We may assume that $\sigma \eta \leqslant 1 / 2$. We set

$$
\delta^{\prime}=\frac{(\eta \sigma)^{2}}{240} \leqslant \frac{1}{3}
$$

and we let $\delta$ be sufficiently small (as given by Fact 1.3 ), so that $\operatorname{DISC}(\delta) \operatorname{implies} \operatorname{DISC}_{2}\left(\delta^{\prime}\right)$. Again, without loss of generality, we may assume $\delta \leqslant \delta^{\prime}$. We let $n_{0} \geqslant 4$ be sufficiently large so that $n \sim_{\delta^{\prime} / 2}(n-1)$ for every $n \geqslant n_{0}$. We shall now show that this choice of constants will do. Thus, let $G=G(\Gamma, A)$ with $|\Gamma|=n \geqslant n_{0}$ and $m>1 / \delta$ be given (we follow the notation in Setup A.5), and suppose that $G$ satisfies $\operatorname{DISC}(\delta)$.

Let $0 \leqslant D_{1}<D_{2} \leqslant\lfloor m / 2\rfloor+1$ be such that $D_{2}-D_{1} \geqslant \eta m$. We fix

$$
d=\left\lfloor\frac{\eta \sigma m}{60}\right\rfloor \geqslant\left\lfloor\frac{\eta \sigma}{60 \delta}\right\rfloor \geqslant\left\lfloor\frac{4}{\eta \sigma}\right\rfloor \geqslant 4,
$$

and

$$
d_{1}=\max \left\{D_{1}, d\right\} \quad \text { and } \quad d_{2}=\min \left\{D_{2},\left\lfloor\frac{m}{2}\right\rfloor-d-1\right\} .
$$

Using $m>1 / \delta \geqslant 1 / \delta^{\prime}=240 /(\eta \sigma)^{2}, 2 \eta \sigma \leqslant 1$, and $d+1 \leqslant 3 d / 2$, we see that

$$
1<\delta m \leqslant \delta^{\prime} m \leqslant \frac{\delta^{\prime} m}{2 \eta \sigma}=\frac{\eta \sigma m}{480} \leqslant d<d+1 \leqslant \frac{\eta \sigma m}{40}
$$

and

$$
D_{2}-D_{1}-2(d+1) \leqslant d_{2}-d_{1} \leqslant D_{2}-D_{1} .
$$

Now we check that the assumptions of Corollary A.16, Lemma A.17, and Lemma A.18 hold simultaneously. It is obvious that the conditions in Lemma A.17 and A.18 hold by our choice of $\delta, \delta^{\prime}, n_{0}$, and the inequalities in (56). Moreover, conditions ( $\left.i i\right)$ and ( $\left.i v\right)$ of Corollary A.16 hold by the definition of $\delta$ (yielding $\operatorname{DISC}_{2}\left(\delta^{\prime}\right)$ for $G$ ), and $n_{0}$. It remains 
to verify $($ iii $)$ and $(i)$ in Corollary A.16. We start with condition $(i i i)$. For this we note that, by the left-hand side of (57) and by (56),

$$
d_{2}-d_{1} \geqslant \eta m-\frac{\eta \sigma m}{20} .
$$

Using $1<\delta m,(56)$, and $\delta \leqslant \delta^{\prime} \leqslant \eta / 6$ (see (55)), we verify (iii):

$$
\begin{aligned}
\frac{1}{2}\left(d_{2}-d_{1}-1\right)-d & \geqslant \frac{1}{2}\left(\eta m-\frac{\eta \sigma m}{20}-\delta m-\frac{\eta \sigma m}{20}\right) \\
& \geqslant \frac{1}{2}\left(\frac{\eta m}{2}-\delta m\right) \geqslant \frac{3 \delta^{\prime} m-\delta^{\prime} m}{2}=\delta^{\prime} m .
\end{aligned}
$$

Moreover, the last inequality implies $d_{2}-1-d_{1}>1$ (using $\delta^{\prime} m>1$ ) and thus $(i)$ of Corollary A.16 follows as well.

Having verified the assumptions of Corollary A.16, Lemma A.17, and Lemma A.18, we use these lemmas to verify the claim of Lemma A.9, i.e.,

$$
\left|A \cap \varrho^{-1}\left(\left[D_{1}, D_{2}\right)\right)\right| \sim_{\sigma} \frac{D_{2}-D_{1}}{m}|A| .
$$

We first derive the upper bound in (58). Note that

$$
\begin{aligned}
\left|A \cap \varrho^{-1}\left(\left[D_{1}, D_{2}\right)\right)\right| \leqslant\left|A \cap \varrho^{-1}([0, d))\right|+\left|A \cap \varrho^{-1}\left(\left[d_{1}, d_{2}\right)\right)\right| \\
+\left|A \cap \varrho^{-1}([\lfloor m / 2\rfloor-d-1,\lfloor m / 2\rfloor+1))\right| .
\end{aligned}
$$

Applying Lemma A.17, Corollary A.16, and Lemma A.18 to the first, second, and third terms, of the right-hand side of the above inequality, respectively, yields

$$
\begin{aligned}
\left|A \cap \varrho^{-1}\left(\left[D_{1}, D_{2}\right)\right)\right| & \leqslant 4 \frac{d}{m}|A|+\left(\frac{|A|}{m}\left(d+1+\frac{\delta^{\prime} m^{2}}{4 d}\right)+\frac{d_{2}-d_{1}}{m}|A|\right)+4 \frac{d+1}{m}|A| \\
& \leqslant \frac{|A|}{m}\left(10(d+1)+\frac{\delta^{\prime} m^{2}}{4 d}\right)+\frac{d_{2}-d_{1}}{m}|A| .
\end{aligned}
$$

Using (55), (56), and (57), we can bound this last expression further by

$$
\frac{|A|}{m}\left(\frac{\sigma \eta m}{4}+\frac{\sigma \eta m}{2}\right)+\frac{D_{2}-D_{1}}{m}|A|,
$$

and, finally, $D_{2}-D_{1} \geqslant \eta m$ gives

$$
\left|A \cap \varrho^{-1}\left(\left[D_{1}, D_{2}\right)\right)\right| \leqslant(1+\sigma) \frac{D_{2}-D_{1}}{m}|A| .
$$

It is left for us to show the lower bound in (58). Note

$$
\left|A \cap \varrho^{-1}\left(\left[D_{1}, D_{2}\right)\right)\right| \geqslant\left|A \cap \varrho^{-1}\left(\left[d_{1}, d_{2}\right)\right)\right|,
$$

and hence Corollary A.16 implies

$$
\left|A \cap \varrho^{-1}\left(\left[D_{1}, D_{2}\right)\right)\right| \geqslant \frac{d_{2}-d_{1}}{m}|A|-\frac{|A|}{m}\left(d+1+\frac{\delta^{\prime} m^{2}}{4 d}\right) .
$$


Similar calculations to the ones above, based on (57), (55), and (56), show that

$$
\begin{aligned}
\frac{d_{2}-d_{1}}{m}|A|-\frac{|A|}{m}\left(d+1+\frac{\delta^{\prime} m^{2}}{4 d}\right) & \geqslant \frac{D_{2}-D_{1}}{m}|A|-\frac{|A|}{m}\left(3(d+1)+\frac{\delta^{\prime} m^{2}}{4 d}\right) \\
& \geqslant \frac{D_{2}-D_{1}}{m}|A|\left(1-\frac{\sigma \eta m}{10\left(D_{2}-D_{1}\right)}-\frac{\sigma \eta m}{2\left(D_{2}-D_{1}\right)}\right) .
\end{aligned}
$$

Again, since $D_{2}-D_{1} \geqslant \eta m$, it follows from (60) combined with (61) that

$$
\left|A \cap \varrho^{-1}\left(\left[D_{1}, D_{2}\right)\right)\right| \geqslant(1-\sigma) \frac{D_{2}-D_{1}}{m}|A| .
$$

Finally, (59) and (62) imply (58), and therefore Lemma A.9 is proved.

A.2.4. $\mathbb{Z}$-INT-DISC implies EIG. In this section we give the proofs of Lemmas A.10 and A.11. We start with the proof of Lemma A.10, which "translates" the results of Lemma A.9 for $\varrho$ and $\mathbb{Z} / m \mathbb{Z}$ to $\chi_{\mathrm{ARG}}$ and $\mathbb{R} / 2 \pi \mathbb{R}$.

Proof of Lemma A.10. Let $\sigma$ and $\eta$ be as in the statement of Lemma A.10, and suppose $A$ satisfies $\mathbb{Z}$-INT-DISC $(\varrho ; \eta / 2, \sigma / 3)$. Let $0 \leqslant \vartheta_{1}<\vartheta_{2} \leqslant \pi$ with $\vartheta_{2}-\vartheta_{1} \geqslant 2 \pi \eta$ be given. Our aim is to show (25), i.e.,

$$
\left|A \cap \chi_{\mathrm{ARG}}^{-1}\left(\left[\vartheta_{1}, \vartheta_{2}\right]\right)\right| \sim_{\sigma} \frac{\vartheta_{2}-\vartheta_{1}}{2 \pi}|A| .
$$

Recall we have $\varrho: \Gamma \rightarrow \mathbb{Z} / m \mathbb{Z}$ and $\Omega: \mathbb{Z} / m \mathbb{Z} \rightarrow \mathbb{R} / 2 \pi \mathbb{R}$ such that $\chi_{\mathrm{ARG}}=\Omega \varrho$ (see Setup A.5). Put

$$
D_{1}=\left\lceil m \frac{\vartheta_{1}}{2 \pi}\right\rceil \text { and } \quad D_{2}=\left\lfloor m \frac{\vartheta_{2}}{2 \pi}\right\rfloor+1
$$

Observe that

$$
\frac{2 \pi}{m}\left(\left\lceil\frac{m \vartheta_{1}}{2 \pi}\right\rceil-1\right)<\vartheta_{1} \leqslant \frac{2 \pi}{m}\left\lceil\frac{m \vartheta_{1}}{2 \pi}\right\rceil
$$

and hence we have

$$
\frac{2 \pi}{m}\left(\left\lceil\frac{m \vartheta_{1}}{2 \pi}\right\rceil-1\right)=\Omega\left(D_{1}-1\right)<\vartheta_{1} \leqslant \Omega\left(D_{1}\right)=\frac{2 \pi}{m}\left\lceil\frac{m \vartheta_{1}}{2 \pi}\right\rceil .
$$

Similarily, one may check that $\Omega\left(D_{2}-1\right) \leqslant \vartheta_{2}<\Omega\left(D_{2}\right)$, and consequently

$$
\Omega^{-1}\left(\left[\vartheta_{1}, \vartheta_{2}\right]\right)=\left[D_{1}, D_{2}\right)
$$

We now observe that

$$
D_{2}-D_{1}=\left\lfloor m \frac{\vartheta_{2}}{2 \pi}\right\rfloor+1-\left\lceil m \frac{\vartheta_{1}}{2 \pi}\right\rceil=\frac{m}{2 \pi}\left(\vartheta_{2}-\vartheta_{1}\right)+O_{1}(1) .
$$

Using that $m \eta \geqslant m \eta \sigma \geqslant 3$, we deduce that

$$
D_{2}-D_{1} \geqslant \frac{m}{2 \pi}\left(\vartheta_{2}-\vartheta_{1}\right)-1 \geqslant \eta m-1 \geqslant \frac{1}{2} \eta m .
$$


Hence, by property $\mathbb{Z}$-INT-DISC $(\varrho ; \eta / 2, \sigma / 3)$, we have

$$
\begin{aligned}
\left|A \cap \chi_{\mathrm{ARG}}^{-1}\left(\left[\vartheta_{1}, \vartheta_{2}\right]\right)\right| & =\left|A \cap \varrho^{-1}\left(\left[D_{1}, D_{2}\right)\right)\right| \\
& \sim_{\sigma / 3} \frac{1}{m}\left(D_{2}-D_{1}\right)|A| \\
& =\frac{1}{m}\left(m \frac{\vartheta_{2}}{2 \pi}-m \frac{\vartheta_{1}}{2 \pi}+O_{1}(1)\right)|A| \\
& =\frac{1}{2 \pi}\left(\vartheta_{2}-\vartheta_{1}+O_{1}\left(\frac{2 \pi}{m}\right)\right)|A|,
\end{aligned}
$$

which, using that $m \eta \sigma \geqslant 3$, is

$$
\frac{1}{2 \pi}\left(1+O_{1}\left(\frac{\sigma}{3}\right)\right)\left(\vartheta_{2}-\vartheta_{1}\right)|A|
$$

We conclude from (63) and (64) that

$$
\left|A \cap \chi_{\mathrm{ARG}}^{-1}\left(\left[\vartheta_{1}, \vartheta_{2}\right]\right)\right| \sim_{\sigma} \frac{\left(\vartheta_{2}-\vartheta_{1}\right)}{2 \pi}|A|,
$$

as required. The proof of Lemma A.10 is complete.

Finally, we prove the last auxiliary lemma, Lemma A.11, used in the proof of the main theorem, Theorem 1.6.

Proof of Lemma A.11. Let $0<\varepsilon \leqslant 1$ be given. We define the constants $\eta$ and $\sigma>0$ as follows.

$$
\eta=\frac{\varepsilon}{8 \pi}<\frac{1}{16} \quad \text { and } \quad \sigma=\frac{1}{8} \varepsilon .
$$

In the remainder of the proof, we show that the above choice for the constants $\eta$ and $\sigma>0$ will do. Thus, let us suppose that the set $A \subseteq \Gamma \backslash\{0\}$ satisfies property $S^{1}$-ARC-DISC $\left(\chi_{\mathrm{ARG}} ; \eta, \sigma\right)$. Our aim is to show that $(26)$ holds, i.e.,

$$
\left|\lambda^{(\chi)}\right|=\left|\sum_{a \in A} \chi(a)\right| \leqslant \varepsilon|A|
$$

For a complex number $z \in \mathbb{C}$, in what follows, we write $\operatorname{Re}(z)$ for the real and $\operatorname{Im}(z)$ for the imaginary part of $z$. Let us first observe that, owing to the fact that the eigenvalues of an undirected graph are real and that $A=-A$, we have

$$
\begin{aligned}
\lambda^{(\chi)} & =\sum_{a \in A} \chi(a)=\operatorname{Re}\left(\sum_{a \in A} \chi(a)\right) \\
& =2 \operatorname{Re}\left(\sum_{a \in A}\{\chi(a): \operatorname{Im}(\chi(a))>0\}\right)+\operatorname{Re}\left(\sum_{a \in A}\{\chi(a): \operatorname{Im}(\chi(a))=0\}\right) .
\end{aligned}
$$


Moreover, it follows from $S^{1}-\mathrm{ARC}-\operatorname{DISC}\left(\chi_{\mathrm{ARG}} ; \eta, \sigma\right)$ that

$$
\begin{aligned}
\left|A \cap \chi^{-1}(1)\right|=\left|A \cap \chi_{\mathrm{ARG}}^{-1}(0)\right| & \leqslant\left|A \cap \chi_{\mathrm{ARG}}^{-1}([0,2 \pi \eta])\right| \\
& \leqslant(1+\sigma) \eta|A| \leqslant 2 \eta|A| \leqslant \frac{1}{4} \varepsilon|A| .
\end{aligned}
$$

Similarly, we observe that $\left|A \cap \chi^{-1}(-1)\right|=\left|A \cap \chi_{\mathrm{ARG}}^{-1}(\pi)\right| \leqslant(1 / 4) \varepsilon|A|$ and thus

$$
\left|\operatorname{Re}\left(\sum_{a \in A}\{\chi(a): \operatorname{Im}(\chi(a))=0\}\right)\right|=|| A \cap \chi^{-1}(1)|-| A \cap \chi^{-1}(-1)|| \leqslant \frac{1}{4} \varepsilon|A| .
$$

Therefore, we infer from (66) that

$$
\left|\lambda^{(\chi)}\right| \leqslant 2\left|\operatorname{Re}\left(\sum_{a \in A}\{\chi(a): \operatorname{Im}(\chi(a)) \geqslant 0\}\right)\right|+\frac{1}{4} \varepsilon|A| .
$$

Thus, we are interested in $\operatorname{Re}(\chi(a))(a \in A, \operatorname{Im}(\chi(a)) \geqslant 0)$.

Put

$$
k=\left\lfloor\frac{\pi / 2}{4 \pi \eta}\right\rfloor=\left\lfloor\frac{1}{8 \eta}\right\rfloor \text { and } \varphi=\frac{\pi}{2 k} .
$$

Observe for later reference that by (65) and (68)

$$
4 \pi \eta \leqslant \varphi \leqslant 8 \pi \eta=\varepsilon .
$$

We shall subdivide the upper half of $S^{1}$ into $2 k$ arcs, symmetric with respect to the imaginary axis (in fact, $\mathbf{i}$ will be left out). The endpoints of the arcs will be $0, \varphi, 2 \varphi, \ldots, \pi$. Let us in fact denote by $I_{j}^{+}$the arc of the $z=\exp (\vartheta \mathbf{i}) \in S^{1}$ with

$$
(j-1) \varphi \leqslant \vartheta<j \varphi
$$

for all $1 \leqslant j \leqslant k$. Similarly, we let $I_{j}^{-}$be the arc of the $z=\exp (\vartheta \mathbf{i}) \in S^{1}$ with

$$
\pi-j \varphi<\vartheta \leqslant \pi-(j-1) \varphi
$$

for all $1 \leqslant j \leqslant k$. Clearly, if $a \in \Gamma$ is such that $\chi(a) \in I_{j}^{+}(1 \leqslant j \leqslant k)$, then

$$
\cos (j \varphi)<\operatorname{Re}(\chi(a)) \leqslant \cos ((j-1) \varphi) .
$$

Similarly, if $a \in \Gamma$ is such that $\chi(a) \in I_{j}^{-}(1 \leqslant j \leqslant k)$, then

$$
-\cos ((j-1) \varphi)<\operatorname{Re}(\chi(a)) \leqslant-\cos (j \varphi) .
$$

We now state and prove the following claim.

Claim A.19. For all $1 \leqslant j \leqslant k$ and both $* \in\{+,-\}$, we have

$$
\left|A \cap \chi^{-1}\left(I_{j}^{*}\right)\right| \sim_{2 \sigma} \frac{\varphi}{2 \pi}|A| .
$$


Proof. This claim follows easily from $S^{1}$-ARC-DISC $\left(\chi_{\mathrm{ARG}} ; \eta, \sigma\right)$. Let $0 \leqslant \psi_{1}<\psi_{2} \leqslant \pi$ be such that

$$
\psi_{2}-\psi_{1} \geqslant 4 \pi \eta
$$

We shall show that

$$
\left|A \cap \chi_{\mathrm{ARG}}^{-1}\left(\left[\psi_{1}, \psi_{2}\right)\right)\right| \sim_{2 \sigma} \frac{1}{2 \pi}\left(\psi_{2}-\psi_{1}\right)|A| .
$$

One may similarly show that

$$
\left|A \cap \chi_{\mathrm{ARG}}^{-1}\left(\left(\psi_{1}, \psi_{2}\right]\right)\right| \sim_{2 \sigma} \frac{1}{2 \pi}\left(\psi_{2}-\psi_{1}\right)|A| .
$$

Claim A.19 follows from (74) and (75). In particular (74) with $\psi_{1}=(j-1) \varphi$ and $\psi_{2}=j \varphi$ gives the claim for intervals of the type $I_{j}^{+}$and similarly (75) yields (72) for intervals of the type $I_{j}^{-}$.

To prove (74), observe first that there is a $\xi>0$ such that

$$
A \cap \chi_{\mathrm{ARG}}^{-1}\left(\left[\psi_{1}, \psi_{2}\right)\right)=A \cap \chi_{\mathrm{ARG}}^{-1}\left(\left[\psi_{1}, \psi_{2}-\xi\right]\right) .
$$

Moreover, we may clearly assume that

$$
\frac{\xi}{4 \pi \eta} \leqslant \frac{\sigma}{2}
$$

Then $\psi_{2}-\psi_{1}-\xi \geqslant 2 \pi \eta$, and hence we may apply $S^{1}$-ARC-DISC $\left(\chi_{\mathrm{ARG}} ; \eta, \sigma\right)$ to deduce that

$$
\left|A \cap \chi_{\mathrm{ARG}}^{-1}\left(\left[\psi_{1}, \psi_{2}-\xi\right]\right)\right| \sim_{\sigma} \frac{1}{2 \pi}\left(\psi_{2}-\psi_{1}-\xi\right)|A|,
$$

and, by (73) and (77), we have

$$
\frac{1}{2 \pi}\left(\psi_{2}-\psi_{1}-\xi\right)|A| \sim_{\sigma / 2} \frac{1}{2 \pi}\left(\psi_{2}-\psi_{1}\right)|A| .
$$

Relation (74) follows from (76), (78), and (79), by

$$
A \cap \chi_{\mathrm{ARG}}^{-1}\left(\left[\psi_{1}, \psi_{2}\right)\right)=A \cap \chi_{\mathrm{ARG}}^{-1}\left(\left[\psi_{1}, \psi_{2}-\xi\right]\right) \sim_{2 \sigma} \frac{1}{2 \pi}\left(\psi_{2}-\psi_{1}\right)|A| .
$$

Claim A.19 and inequalities (70) and (71) may now be used to estimate the sum in (67). We have

$$
\operatorname{Re}\left(\sum_{a \in A}\{\chi(a): \operatorname{Im}(\chi(a)) \geqslant 0\}\right)=\operatorname{Re}\left(\sum_{j=1}^{k} \sum_{a \in A}\left\{\chi(a): \chi(a) \in I_{j}^{+} \cup I_{j}^{-}\right\}\right) .
$$

Fix $1 \leqslant j \leqslant k$. We have, by Claim A.19 and inequalities (70) and (71),

$$
\operatorname{Re}\left(\sum_{a \in A}\left\{\chi(a): \chi(a) \in I_{j}^{+} \cup I_{j}^{-}\right\}\right) \leqslant \frac{\varphi}{2 \pi}|A|((1+2 \sigma) \cos ((j-1) \varphi)-(1-2 \sigma) \cos (j \varphi)) .
$$


Using that $\cos ((j-1) \varphi)-\cos (j \varphi) \leqslant \varphi$, we observe that the right-hand side of $(81)$ is smaller than

$$
\frac{\varphi}{2 \pi}|A|(\varphi+4 \sigma)
$$

Therefore, from (81) and (82) we deduce that the expression in (80) is, in absolute value, at most

$$
k \frac{\varphi}{2 \pi}|A|(\varphi+4 \sigma)=\frac{1}{4}|A|(\varphi+4 \sigma) \leqslant \frac{1}{4}|A|\left(\varepsilon+\frac{1}{2} \varepsilon\right)=\frac{3}{8} \varepsilon|A|,
$$

where in this inequality we used (65) and (69). Combining (83) with (67), we infer that

$$
\left|\lambda^{(\chi)}\right| \leqslant 2 \frac{3}{8} \varepsilon|A|+\frac{1}{4} \varepsilon|A|=\varepsilon|A|,
$$

as claimed in Lemma A.11.

\section{REFERENCES}

[1] N. Alon, Eigenvalues and expanders, Combinatorica 6 (1986), no. 2, 83-96, DOI 10.1007/BF02579166. Theory of computing (Singer Island, Fla., 1984). MR875835 11

[2] N. Alon and F. R. K. Chung, Explicit construction of linear sized tolerant networks, Discrete Math. 72 (1988), no. 1-3, 15-19, DOI 10.1016/0012-365X(88)90189-6. MR975519 11

[3] N. Alon and V. D. Milman, $\lambda_{1}$, isoperimetric inequalities for graphs, and superconcentrators, J. Combin. Theory Ser. B 38 (1985), no. 1, 73-88, DOI 10.1016/0095-8956(85)90092-9. MR782626 11

[4] N. Alon, A. Coja-Oghlan, H. Hàn, M. Kang, V. Rödl, and M. Schacht, Quasi-randomness and algorithmic regularity for graphs with general degree distributions, SIAM J. Comput. 39 (2010), no. 6, 2336-2362, DOI 10.1137/070709529. MR2644348 ^1

[5] N. Alon and J. H. Spencer, The probabilistic method, 3rd ed., Wiley-Interscience Series in Discrete Mathematics and Optimization, John Wiley \& Sons, Inc., Hoboken, NJ, 2008. With an appendix on the life and work of Paul Erdős. MR2437651 $\uparrow 2.2$

[6] L. Babai, Spectra of Cayley graphs, J. Combin. Theory Ser. B 27 (1979), no. 2, 180-189, DOI 10.1016/0095-8956(79)90079-0. MR546860 22.1

[7] Y. Bilu and N. Linial, Lifts, discrepancy and nearly optimal spectral gap, Combinatorica 26 (2006), no. 5, 495-519, DOI 10.1007/s00493-006-0029-7. MR2279667 $\uparrow 1$

[8] F. Chung and R. Graham, Sparse quasi-random graphs, Combinatorica 22 (2002), no. 2, 217-244, DOI 10.1007/s004930200010. Special issue: Paul Erdős and his mathematics. MR1909084 ^1, 1, 1

[9] F. R. K. Chung, R. L. Graham, and R. M. Wilson, Quasi-random graphs, Combinatorica 9 (1989), no. 4, 345-362, DOI 10.1007/BF02125347. MR1054011 11

[10] D. Conlon, J. Fox, and Y. Zhao, Extremal results in sparse pseudorandom graphs, Adv. Math. 256 (2014), 206-290, DOI 10.1016/j.aim.2013.12.004. MR3177293 ^1

[11] D. Conlon and Y. Zhao, Quasirandom Cayley graphs, available at arXiv:1603.03025. Submitted. $\uparrow 1$

[12] W. E. Donath and A. J. Hoffman, Algorithms for partitioning of graphs and computer logic based on eigenvectors of connection matrices, IBM Techn. Disclosure Bull. 15 (1972), 938-944. $\uparrow 1$

[13] W. E. Donath and A. J. Hoffman, Lower bounds for the partitioning of graphs, IBM J. Res. Develop. 17 (1973), 420-425. MR0329965 $\uparrow 1$ 
[14] M. Fiedler, Algebraic connectivity of graphs, Czechoslovak Math. J. 23(98) (1973), 298-305. MR0318007 $\uparrow 1$

[15] _ A property of eigenvectors of nonnegative symmetric matrices and its application to graph theory, Czechoslovak Math. J. 25(100) (1975), no. 4, 619-633. MR0387321 $\uparrow 1$

[16] P. Frankl, V. Rödl, and R. M. Wilson, The number of submatrices of a given type in a Hadamard matrix and related results, J. Combin. Theory Ser. B 44 (1988), no. 3, 317-328, DOI 10.1016/00958956(88)90040-8. MR941440 $\uparrow 1$

[17] W. T. Gowers, personal communication. $\uparrow 1$

[18] K. M. Hall, R-Dimensional quadratic placement algorithm, Management Science Series A (Theory) 17 (1970), no. 3, 219-229 (English). $\uparrow 1$

[19] Y. Kohayakawa and V. Rödl, Regular pairs in sparse random graphs. I, Random Structures Algorithms 22 (2003), no. 4, 359-434, DOI 10.1002/rsa.10081. MR1980964 ^1

[20] Y. Kohayakawa, V. Rödl, and P. Sissokho, Embedding graphs with bounded degree in sparse pseudorandom graphs, Israel J. Math. 139 (2004), 93-137, DOI 10.1007/BF02787543. MR2041225 ^1

[21] M. Krivelevich and B. Sudakov, Pseudo-random graphs, More sets, graphs and numbers, Bolyai Soc. Math. Stud., vol. 15, Springer, Berlin, 2006, pp. 199-262, DOI 10.1007/978-3-540-32439-3_10. MR2223394 $\uparrow 1,1$

[22] L. Lovász, Spectra of graphs with transitive groups, Period. Math. Hungar. 6 (1975), no. 2, 191-195. MR0398886 $\uparrow 2.1$

[23] L. Lovász, Combinatorial problems and exercises, 2nd ed., AMS Chelsea Publishing, Providence, RI, 2007. MR2321240 $\uparrow 2.1$

[24] V. Rödl, On universality of graphs with uniformly distributed edges, Discrete Math. 59 (1986), no. 1-2, 125-134, DOI 10.1016/0012-365X(86)90076-2. MR837962 ^1

[25] J.-P. Serre, Linear representations of finite groups, Springer-Verlag, New York-Heidelberg, 1977. Translated from the second French edition by Leonard L. Scott; Graduate Texts in Mathematics, Vol. 42. MR0450380 $\uparrow 2.1$

[26] A. Sinclair and M. Jerrum, Approximate counting, uniform generation and rapidly mixing Markov chains, Inform. and Comput. 82 (1989), no. 1, 93-133, DOI 10.1016/0890-5401(89)90067-9. MR1003059 $\uparrow 1$

[27] D. Spielman, Spectral graph theory, Combinatorial scientific computing, Chapman \& Hall/CRC Comput. Sci. Ser., CRC Press, Boca Raton, FL, 2012, pp. 495-524, DOI 10.1201/b11644-19. MR2952760 ^1

[28] D. A. Spielman and S.-H. Teng, Spectral partitioning works: planar graphs and finite element meshes, Linear Algebra Appl. 421 (2007), no. 2-3, 284-305, DOI 10.1016/j.laa.2006.07.020. MR2294342 11

[29] R. M. Tanner, Explicit concentrators from generalized N-gons, SIAM J. Algebraic Discrete Methods 5 (1984), no. 3, 287-293, DOI 10.1137/0605030. MR752035 ^1

[30] A. Thomason, Pseudorandom graphs, Random graphs '85 (Poznań, 1985), North-Holland Math. Stud., vol. 144, North-Holland, Amsterdam, 1987, pp. 307-331. MR930498 11

[31] _ Random graphs, strongly regular graphs and pseudorandom graphs, Surveys in combinatorics 1987 (New Cross, 1987), London Math. Soc. Lecture Note Ser., vol. 123, Cambridge Univ. Press, Cambridge, 1987, pp. 173-195. MR905280 ^1 
Instituto de Matemática e Estatística, Universidade de São Paulo, São Paulo, Brazil E-mail address: yoshi@ime.usp.br

Department of Mathematics and Computer Science, Emory University, Atlanta, USA

E-mail address: rodl@mathcs.emory.edu

Fachbereich Mathematik, Universität Hamburg, Hamburg, Germany

E-mail address: schacht@math.uni-hamburg.de 